\title{
SOME PRODUCT FORMULAE FOR NONSIMPLY CONNECTED SURGERY PROBLEMS
}

\author{
R. J. MILGRAM AND ANDREW RANICKI
}

\begin{abstract}
For an $n$-dimensional normal map $f: M^{n} \rightarrow N^{n}$ with finite fundamental group $\pi_{1}(N)=\pi$ and PL 1 torsion kernel $Z[\pi]$-modules $K_{*}(M)$ the surgery obstruction $\sigma_{*}(f) \in L_{n}^{h}(Z[\pi])$ is expressed in terms of the projective classes $\left[K_{*}(M)\right] \in \tilde{K}_{0}(Z[\pi])$, assuming $K_{i}(M)=0$ if $n=2 i$. This expression is used to evaluate in certain cases the surgery obstruction $\sigma_{*}(g) \in L_{m+n}^{h}\left(Z\left[\pi_{1} \times \pi\right]\right)$ of the $(m+n)$-dimensional normal map $g=1 \times f: M_{1} \times M \rightarrow M_{1} \times N$ defined by product with an $m$-dimensional manifold $M_{1}$, where $\pi_{1}=\pi_{1}\left(M_{1}\right)$.
\end{abstract}

A key problem in surgery theory is to understand how to calculate the surgery obstructions for surgery problems

$$
f: M^{n} \rightarrow N^{n}
$$

where $f$ is a degree 1 normal map and $M^{n}, N^{n}$ are closed $n$-dimensional manifolds. C. T. C. Wall [20] has pointed out that the problem $(*)$ determines an element

$$
\alpha(f) \in \Omega_{n}\left(B_{\pi_{1}(N)} \times G / \text { TOP }, B_{\pi_{1}(N)} \times\{\text { pt. }\}\right)
$$

and there is a map

$$
e: \Omega_{*}\left(B_{\pi_{1}(N)} \times G / \mathrm{TOP}, B_{\pi_{1}(N)} \times\{\mathrm{pt} .\}\right) \rightarrow L_{*}\left(\pi_{1}(N)\right)
$$

so that the surgery obstruction $\sigma_{*}(f)$ is $e(\alpha(f))$.

Wall also pointed out that if $\pi_{1}(N)$ is finite, then $\alpha(f)$ is already determined by restriction to the 2-Sylow subgroup of $\pi_{1}$, and the groups $L_{*}(\pi)$ have been extensively studied when $\pi$ is a finite 2-group. (See e.g. Pardon [12], CarlssonMilgram [3, 5], Hambleton-Milgram [9], Wall [21] and Bak-Kolster [1].) Indeed the projective $L$-groups $L_{*}^{p}(\pi)$ are completely known, and the groups $L_{*}^{h}(\pi)$ and $L_{*}^{s}(\pi)$ are effectively computable in tems of certain additional facts about $\tilde{K}_{0}(Z[\pi])$ and $W h(\pi)$.

So further progress depends on studying the map $e$. For the projective $L$ groups $L_{*}^{p}(\pi)$ with $\pi$ a finite 2-group, this was done by L. Taylor-B. Williams [18] and independently by $\mathrm{I}$. Hambleton [8]. But for the more basic case of $L_{*}^{h}(\pi)$ our information is much more limited. There are some key examples (Cappell-Shaneson [2], Taylor-Williams [18]) which show that this problem is much harder, but general information is hard to come by.

Received by the editors June 3, 1985.

1980 Mathematics Subject Classification (1985 Revision). Primary 57R67. 
In this note we initiate an attack on this problem from a different direction, that of product formulae for surgery obstructions.

Under certain circumstances we demonstrate the existence of "semicharacteristic classes" and relate the surgery obstructions of surgery problems of the form

$$
\text { id } \times f: M_{1} \times M \rightarrow M_{1} \times N
$$

with $\pi_{1}\left(M_{1}\right)=\pi_{1}$ and $\pi_{1}(N)=\pi$ to a certain product $\chi_{1 / 2}\left(M_{1}\right) \cdot \operatorname{tr}\left(\sigma_{*}(f)\right)$ in $L_{*}^{h}\left(\pi_{1} \times \pi\right)$, or when appropriate simplifying conditions are not present, to a formula only slightly more complicated (Theorems 3.3 and 3.4).

These results are applied in $\$ 4$ to give a direct proof of the Morgan-Pardon, Taylor-Williams result for the group $\pi=Z / 2 \times Z / 4$, which is the simplest case in which a surgery obstruction occurs which is trivial in $L_{*}^{p}(\pi)$ but not in $L_{*}^{h}(\pi)$, and provide the main intuitions for the key step in [10], where ideas of Clauwens [6] are used to completely classify surgery obstructions for closed manifolds with finite fundamental group crossed with the Kervaire problem.

We thank Ian Hambleton for a careful reading of a preliminary version and some valuable comments.

1. Evaluating the surgery obstruction. In this section we study the question of when the surgery obstruction $\sigma_{*}(f) \in L_{n}(Z[\pi])$ of an $n$-dimensional normal map $f$ : $M \rightarrow N$ with $\pi_{1}(N)=\pi$ is determined only by the kernel $Z[\pi]$-modules

$$
K_{*}(M)=\operatorname{ker}\left(\tilde{f}_{*}: H_{*}(\tilde{M}) \rightarrow H_{*}(\tilde{N})\right) \text {. }
$$

EXAMPLE 1.0. If $K_{*}(M)=0$, then $f$ is normal bordant to a homotopy equivalence and $\sigma_{*}(f)=0 \in L_{n}^{h}\left(Z[\pi]\right.$ ). (If also $\tau_{1}(M)=\pi_{1}(N)=\pi$, then $f$ is a homotopy equivalence).

Our main tool will be the Rothenberg exact sequence of Ranicki [13] relating the free $L$-groups $L_{*}^{h}(Z[\pi])$ to the projective $L$-groups $L_{*}^{p}(Z[\pi])$ and the projective class group $\tilde{K}_{0}(Z[\pi])$

$$
\begin{aligned}
\cdots \rightarrow \hat{H}_{n}\left(Z_{2}, \tilde{K}_{0}(Z[\pi])\right) \stackrel{\partial}{\rightarrow} L_{n}^{h}(Z[\pi]) & \rightarrow L_{n}^{p}(Z[\pi]) \\
& \rightarrow \hat{H}_{n-1}\left(Z_{2}, \tilde{K}_{0}(Z[\pi])\right) \rightarrow \cdots .
\end{aligned}
$$

EXAMPLE 1.2. If the kernel modules $K_{*}(M)$ are f.g. projective, with $K_{i}(M)=0$ when $n=2 i$, then

$$
\sigma_{*}(f)=\partial\left(\sum_{j<n / 2}(-)^{j}\left[K_{j}(M)\right]\right) \in \operatorname{im}\left(\partial: \hat{H}_{n}\left(Z_{2}, \tilde{K}_{0}(Z[\pi])\right) \rightarrow L_{n}^{h}(Z[\pi])\right) .
$$

(See Corollary 2.3 for a proof.)

We shall obtain an analogous result for normal maps $f: M^{n} \rightarrow N^{n}$ of closed oriented manifolds with finite fundamental group $\pi_{1}(N)=\pi$, such that the kernel modules $K_{*}(M)$ are torsion of projective length 1 . In Theorems 1.11 and 1.14 we shall prove that the surgery obstruction of such a normal map with simply-connected Kervaire invariant 0 if $n=4 k+2$ and $K_{i}(M)=0$ if $n=2 i+1$ is given by

$$
\sigma_{*}(f)=\partial\left(\sum_{j<n / 2}(-)^{j} \chi\left(K_{j}(M)\right)\right) \in \operatorname{im}\left(\partial: \hat{H}_{n}\left(Z_{2}, \tilde{K}_{0}(Z[\pi])\right) \rightarrow L_{n}^{h}(Z[\pi])\right)
$$

with $\chi\left(K_{j}(M)\right) \in \tilde{K}_{0}(Z[\pi])$ the projective characteristic. 
We shall be making extensive use of the commutative braid of exact sequences (1.3)

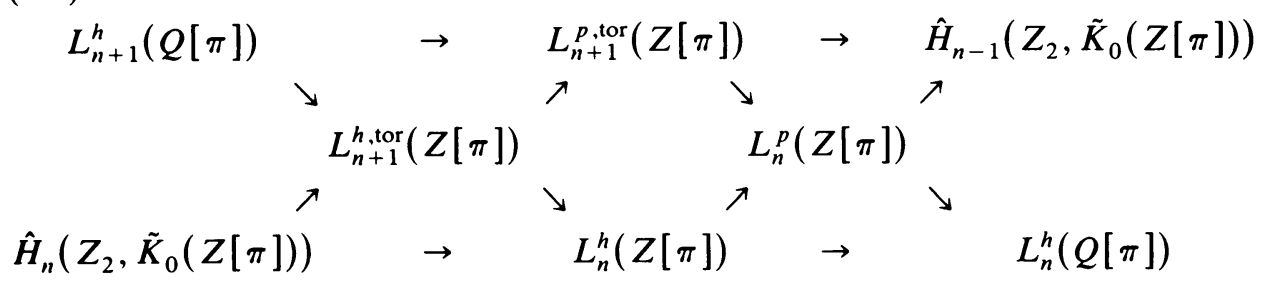

incorporating (1.1) and the localization exact sequences of Pardon [11], Ranicki [16], Carlsson-Milgram [4]

$$
\begin{gathered}
\cdots \rightarrow L_{n+1}^{p, t o r}(Z[\pi]) \rightarrow L_{n}^{p}(Z[\pi]) \rightarrow L_{n}^{h}(Q[\pi]) \rightarrow L_{n}^{p, \text { tor }}(Z[\pi]) \rightarrow \cdots, \\
\cdots \rightarrow L_{n+1}^{h, \text { tor }}(Z[\pi]) \rightarrow L_{n}^{h}(Z[\pi]) \rightarrow L_{n}^{h}(Q[\pi]) \rightarrow L_{n}^{h, \text { tor }}(Z[\pi]) \rightarrow \cdots,
\end{gathered}
$$

as well as the analogue of (1.1) for the torsion $L$-groups

$$
\begin{aligned}
\cdots \rightarrow \hat{H}_{n}\left(Z_{2}, \tilde{K}_{0}(Z[\pi])\right) \stackrel{\partial^{\text {tor }}}{\rightarrow} L_{n+1}^{h, \text { tor }}(Z[\pi]) & \rightarrow L_{n+1}^{p, \text { tor }}(Z[\pi]) \\
& \rightarrow \hat{H}_{n-1}\left(Z_{2}, \tilde{K}_{0}(Z[\pi])\right) \rightarrow \cdots .
\end{aligned}
$$

The inclusion $Z[\pi] \rightarrow Q[\pi]$ induces maps $L_{*}(Z[\pi]) \rightarrow L_{*}^{h}(Q[\pi])$ from the projective $L$-groups of $Z[\pi]$ to the free $L$-groups of $Q[\pi]$, since for every f.g. projective $Z[\pi]$-module $P$ the induced $Q[\pi]$-module $Q \otimes_{Z} P$ is f.g. free, by the theorem of Swan.

The action of $Z_{2}$ on $\tilde{K}_{0}(Z[\pi])$ is by the duality involution

$$
\text { * : } \left.\tilde{K}_{0}(Z[\pi]) \rightarrow \tilde{K}_{0}(Z[\pi]) ; P\right] \rightarrow\left[P^{*}\right],
$$

using the involution on $Z[\pi]$

$$
\because Z[\pi] \rightarrow Z[\pi] ; a=\sum_{g \in \pi} n_{g} g \rightarrow \bar{a}=\sum_{g \in \pi} n_{g} g^{-1}
$$

to define a left $Z[\pi]$-action on the dual f.g. projective $Z[\pi]$-module

$$
P^{*}=\operatorname{Hom}_{Z[\pi]}(P, Z[\pi])
$$

of a f.g. projective left $Z[\dot{\pi}]$-module $P$ by

$$
Z[\pi] \times P^{*} \rightarrow P^{*} ;(a, \phi) \rightarrow(x \rightarrow \phi(x) \bar{a}) .
$$

Thus the homology $Z_{2}$-groups appearing in (1.1) and (1.3) are given (as usual) by

$$
\hat{H}_{n}\left(Z_{2}, \tilde{K}_{0}(Z[\pi])\right)=\frac{\left\{[P] \in \tilde{K}_{0}(Z[\pi]) \mid\left[P^{*}\right]+(-)^{n}[P]=0\right\}}{\left\{[Q]-(-)^{n}\left[Q^{*}\right] \mid[Q] \in \tilde{K}_{0}(Z[\pi])\right\}} .
$$

The even-dimensional $L$-group $L_{2 i}(Z[\pi])$ (resp. $\left.L_{2 i}^{h}(Z[\pi])\right)$ is the Witt group of nonsingular $(-)^{i}$-quadratic forms

$$
\left(K, \lambda: K \times K \rightarrow Z[\pi], \mu: K \rightarrow Q_{(-)} i(Z[\pi])=Z[\pi] /\left\{a-(-)^{i} \bar{a} \mid a \in Z[\pi]\right\}\right)
$$

on f.g. projective (resp. free) $Z[\pi]$-modules $K$. Nonsingular means that the adjoint of $\lambda$ is a $Z[\pi]$-module isomorphism

$$
A \lambda: K \stackrel{\sim}{\rightarrow} K^{*} ; x \rightarrow(y \rightarrow \lambda(x, y)) .
$$


Given a f.g. projective $Z[\pi]$-module $L$, there is defined a hyperbolic nonsingular $(-)^{i}$-quadratic form with lagrangian (= subkernel) $L$

$$
\begin{aligned}
H_{(-)} i(L)=( & L \oplus L^{*}, \lambda: L \oplus L^{*} \rightarrow Z[\pi] ;((x, \phi),(y, \theta)) \\
& \left.\rightarrow \phi(y)+(-)^{i} \overline{\theta(x)}, \mu: L \oplus L^{*} \rightarrow Q_{(-)} i(Z[\pi]) ;(x, \phi) \rightarrow \phi(x)\right)
\end{aligned}
$$

such that $H_{(-)} i(L)=0 \in L_{2 i}^{p}(Z[\pi])$ (resp. $=0 \in L_{2 i}^{h}(Z[\pi])$ if $L$ is free). For $n=2 i$ the map $\partial$ in (1.1) is given by

$$
\text { ə: } \hat{H}_{2 i}\left(Z_{2}, \tilde{K}_{0}(Z[\pi])\right) \rightarrow L_{2 i}^{h}(Z[\pi]) ;[L] \rightarrow H_{(-)} i(L) .
$$

REMARK 1.4. An element $x \in L_{2 i}^{h}(Z[\pi])$ is the image $\partial(y)$ of the element $y \in \hat{H}_{2 i}\left(Z_{2}, \tilde{K}_{0}(Z[\pi])\right)$ if and only if $x$ is represented by a $(-)^{i}$-quadratic form $(K, \lambda, \mu)$ on a f.g. free $Z[\pi]$-module $K$ which admits a f.g. projective lagrangian $L \subset K$ such that

$$
[L]=y \in \hat{H}_{2 i}\left(Z_{2}, \tilde{K}_{0}(Z[\pi])\right) \text {. }
$$

The odd-dimensional $L$-group $L_{2 i+1}^{p}(Z[\pi])$ (resp. $\left.L_{2 i+1}^{h}(Z[\pi])\right)$ is the Witt group of $(-)^{i}$-quadratic formations $(K, \lambda, \mu ; F, G)$, with $(K, \lambda, \mu)$ a nonsingular $(-)^{i}$ quadratic form and $F, G$ projective (resp. free) lagrangians. For $n=2 i+1$ the map $\partial$ in 1.2 is given by

$$
\begin{gathered}
\text { ə: } \hat{H}_{2 i+1}\left(Z_{2}, \tilde{K}_{0}(Z[\pi])\right) \rightarrow L_{2 i+1}^{h}(Z[\pi]) ; \\
{[P] \rightarrow\left(H_{(-)} i(P \oplus-P) ; P \oplus-P, P \oplus(-P)^{*}\right)}
\end{gathered}
$$

with $P,-P$ f.g. projective $Z[\pi]$-modules such that $P \oplus(-P)$ and $P \oplus(-P)^{*}$ are f.g. free $Z[\pi]$-modules.

A $Z[\pi]$-module $M$ is said to have projective length 1 (PL 1 for short) if it admits a f.g. projective $Z[\pi]$-module resolution $0 \rightarrow P_{1} \rightarrow P_{0} \rightarrow M \rightarrow 0$. We shall be primarily concerned with PL 1 torsion $Z[\pi]$-modules $M$, assuming throughout that $\pi$ is a finite group.

REMARK 1.5. If $M$ is a f.g. torsion $Z[\pi]$-module such that $(|M|,|\pi|)=1$, then $M$ is PL 1. (See e.g. Carlsson-Milgram [3].)

A $Z[\pi]$-module $M$ is said to have free length 1 (FPL 1 for short) if it admits a f.g. free $Z[\pi]$-module resolution $0 \rightarrow F_{1} \rightarrow F_{0} \rightarrow M \rightarrow 0$. A PL 1 module $M$ has a projective characteristic

$$
\chi(M)=\left[P_{0}\right]-\left[P_{1}\right] \in \tilde{K}_{0}(Z[\pi]) .
$$

such that $M$ is FPL 1 if and only if $\chi(M)=0$.

The torsion $L$-groups $L_{*}^{p \text {.tor }}(Z[\pi])$ (resp. $\left.L_{*}^{h, \text { tor }}(Z[\pi])\right)$ are the Witt groups of \pm quadratic structures on PL 1 (resp. FPL 1 ) torsion $Z[\pi]$-modules.

The torsion dual of a PL 1 torsion $Z[\pi]$-module $M$ is the PL 1 torsion $Z[\pi]$-module defined by

$$
M^{\wedge}=\operatorname{Hom}_{Z[\pi]}(M, Q[\pi] / Z[\pi]),
$$

with

$$
Z[\pi] \times M^{\wedge} \rightarrow M^{\wedge} ;(a, \phi) \rightarrow(x \rightarrow \phi(x) \bar{a}) .
$$


The dual of a f.g. projective resolution of $M$

$$
0 \rightarrow P_{1} \stackrel{d}{\rightarrow} P_{0} \stackrel{e}{\rightarrow} M \rightarrow 0
$$

is the f.g. projective resolution of $M^{\wedge}$

$$
0 \rightarrow P_{0}^{*} \stackrel{d^{*}}{\rightarrow} P_{1}^{*} \stackrel{e^{\wedge}}{\rightarrow} M^{\wedge} \rightarrow 0
$$

with

$$
\begin{aligned}
e^{\wedge}: P_{1}^{*} \rightarrow M^{\wedge} ; \phi \rightarrow(e(x) \rightarrow & \left.\frac{\theta(y)}{s}\right) \\
& \left(x \in P_{0}, y \in P_{1}, s \in Z-\{0\}, s x=d y \in P_{0}\right) .
\end{aligned}
$$

The projective characteristic of the torsion dual is thus given by

$$
\chi\left(M^{\wedge}\right)=\left[P_{1}^{*}\right]-\left[P_{0}^{*}\right]=-\chi(M)^{*} \in \tilde{K}_{0}(Z[\pi]) .
$$

The even-dimensional torsion $L$-group $L_{2 i}^{p}{ }^{\text {tor }}(Z[\pi])$ (resp. $L_{2 i}^{h \text {,tor }}(Z[\pi])$ ) is the Witt group of nonsingular $(-)^{i}$-quadratic linking forms

$$
\left(K, \lambda: K \times K \rightarrow Q[\pi] / Z[\pi], \mu: K \rightarrow Q_{(-)} i(Q[\pi] / Z[\pi])\right)
$$

on PL 1 (resp. FPL 1) torsion $Z[\pi]$-modules $K$. Nonsingular means that the adjoint of $\lambda$ defines a $Z[\pi]$-module isomorphism

$$
A \lambda: \stackrel{\sim}{\rightarrow} K^{\wedge} ; x \rightarrow(y \rightarrow \lambda(x, y)) .
$$

In order to describe the map

$$
\partial^{\text {tor }:} \hat{H}_{2 i-1}\left(Z_{2}, \tilde{K}_{0}(Z[\pi])\right) \rightarrow L_{2 i}^{h, \text { tor }}(Z[\pi])
$$

appearing in (1.3), note first that for any f.g. projective $Z[\pi]$-module $P$ there exists a PL 1 torsion $Z[\pi]$-module $L$ such that

$$
\chi(L)=[P] \in \tilde{K}_{0}(Z[\pi])
$$

since $Q \otimes_{Z} P$ is a f.g. free $Q[\pi]$-module by Swan's theorem, so that there exists an integral lattice $Z[\pi]^{m} \subset P$ and $L=P / Z[\pi]^{m}$ will do. Now for any PL 1 torsion $Z[\pi]$-module $L$ there is defined a hyperbolic $(-)^{i}$-quadratic linking form with lagrangian $L$

$$
H_{(-)}^{\text {tor }} i(L)=\left(L \oplus L^{\wedge}, \lambda=\left(\begin{array}{cc}
0 & 1 \\
(-)^{i} & 0
\end{array}\right), \mu=\left(\begin{array}{ll}
0 & 1 \\
0 & 0
\end{array}\right)\right)
$$

exactly as the hyperbolic form $H_{(-)} i(P)=\left(P \oplus P^{*}, \lambda, \mu\right)$ but with the torsion dual $L^{\wedge}$ in place of the projective dual $P^{*}$, such that $H_{(-)}^{\text {tor }} i(L)=0 \in L_{2 i}^{p \text {,tor }}(Z[\pi])$ (resp. $H_{(-)}^{\text {tor }} i(L)=0 \in L_{2 i}^{h \text {,tor }}(Z[\pi])$ if $L$ is FPL 1$)$. The map $\partial^{\text {tor }}$ is given by

$$
\partial^{\text {tor }:} \hat{H}_{2 i-1}\left(Z_{2}, \tilde{K}_{0}(Z[\pi])\right) \rightarrow L_{2 i}^{h \text { tor }}(Z[\pi]) ;[P] \rightarrow H_{(-)}^{\text {tor }} i(L),
$$

with $L$ any PL 1 torsion $Z[\pi]$-module such that $\chi(L)=[P] \in \tilde{K}_{0}(Z[\pi])$. The torsion analogue of Remark 1.4 holds:

ReMARK 1.6. An element $x \in L_{2 i}^{h, \text { tor }}(Z[\pi])$ is the image $\partial^{\text {tor }}(y)$ of $y \in$ $\hat{H}_{2 i-1}\left(Z_{2}, \tilde{K}_{0}(Z[\pi])\right)$ if and only if $x$ is represented by a $(-)^{i}$-quadratic linkin form $(K, \lambda, \mu)$ on an FPL 1 torsion $Z[\pi]$-module $K$ which admits a PL 1 torsior lagrangian $L \subset K$ such that

$$
\chi(L)=y \in \hat{H}_{2 i-1}\left(Z_{2}, \tilde{K}_{0}(Z[\pi])\right) .
$$


The odd-dimensional torsion $L$-group $L_{2 i+1}^{p, \text { tor }}(Z[\pi])$ (resp. $\left.L_{2 i+1}^{h \text { tor }}(Z[\pi])\right)$ is essentially the Witt group of nonsingular $(-)^{i}$-quadratic linking formations $(K, \lambda, \mu$; $F, G$ ), with $F, G$ PL 1 (resp. FPL 1) torsion lagrangians of the (-) ${ }^{i}$-quadratic linking form $(K, \lambda, \mu)$, together with some extra structure needed to capture the Kervaire invariant in $L_{2}(Z)$ - the precise definition need not detain us here.

Explicit calculations for the groups and the sequences in (1.3) are made in Carlsson-Milgram [5] and Hambleton-Milgram [9]. Indeed, from [5] we have

LEMMA 1.7. For any finite 2-group $\pi$

$$
L_{1}^{\text {tor, } p}(Z \pi)=(Z / 2)^{c}, \quad L_{3}^{\text {tor, } p}(Z \pi)=L_{3}^{\text {tor } h}\left(\hat{Z}_{2} \pi\right)=(Z / 2)^{d}
$$

where $c, d$ are explicit functions of the rational group ring $Q \pi$.

A nontrivial element in $L_{3}^{\text {tor. } p}(Z \pi)$ is sent by $\partial$ to the nontrivial $Z / 2$ in $L_{2}^{p}(Z)=L_{2}^{h}(Z)=Z / 2$. Recall that the $Z / 2$ in $L_{2}^{h}(Z)$ is the Kervaire invariant, and that the identification of the set of degree-one normal maps $f: M^{n} \rightarrow N^{n}$ with the set of homotopy classes of maps $[N, G / C A T]$ allows one to get an explicit formula for the Kerviare obstruction. Indeed, from the work of Rourke and Sullivan [17], (see in particular Wall [20]), we have that there exists classes $k_{4 i+2} \in$ $H^{4 i+2}(G / C A T, Z / 2)$ so that

$$
\sigma_{*}(f)=\left\langle V^{2} \sum_{i=0}^{n} f^{*}\left(k_{4 i+2}\right),[M]\right\rangle \in Z_{2}
$$

where $\sigma_{*}(f)$ is the Kervaire invariant of the surgery problem induced by the map $f$ : $M \rightarrow G / C A T$. (Here, $V$ is the total Wu class of $M$.)

A. Surgery below the middle dimension.

Proposition 1.9. Let $f: M^{n} \rightarrow N^{n}$ be a normal map of closed n-dimensional manifolds with $\pi_{1}(N)=\pi$ finite, such that the kernel $Z[\pi]$-modules $K_{*}(M)$ are $P L 1$ torsion. For each $j<n / 2, f$ is normal bordant to a $(j-1)$-connected normal map $f^{(j)}: M^{(j)} \rightarrow N$ with kernel modules

$$
K_{r}\left(M^{(j)}\right)=\left(\begin{array}{ll}
0 & \text { if } r<j \text { or } r>n-j, \\
P_{j} \oplus K_{j}(M) & \text { if } r=j, \\
K_{r}(M) & \text { if } j<r<n-j, \\
P_{j}^{*} & \text { if } r=n-j,
\end{array}\right.
$$

where $P_{j}$ is a f.g. projective $Z[\pi]$-module such that

$$
\left[P_{i j}\right]=(-)^{j}\left(\sum_{r<j}(-)^{r} \chi\left(K_{r}(M)\right)\right) \in \tilde{K}_{0}(Z[\pi]) .
$$

Proof. We shall make repeated use of the next lemma, whose proof is obvious.

LEMMA 1.10. Given a f.g. projective $Z[\pi]$-module $P$ and a PL 1 torsion $Z[\pi]$-module $K$, there exists a surjection of a f.g. free $Z[\pi]$-module $F$

$$
\left(\begin{array}{l}
c \\
e
\end{array}\right): F=Z[\pi]^{m} \rightarrow P \oplus K,
$$


in which case $Q=\operatorname{ker}\left(\left(\begin{array}{c}c \\ e\end{array}\right): F \rightarrow P \oplus K\right)$ is a f.g. projective $Z[\pi]$-module with a short exact sequence

$$
0 \rightarrow Q \stackrel{d}{\rightarrow} F \stackrel{(c)}{\rightarrow} P \oplus K \rightarrow 0
$$

such that

$$
\chi(K)+[P]+[Q]=0 \in \tilde{K}_{0}(Z[\pi]) .
$$

The torsion dual $K^{\wedge}$ fits into the exact sequence

$$
0 \rightarrow P^{*} \stackrel{c^{*}}{\rightarrow} F^{*} \stackrel{d^{*}}{\rightarrow} Q^{*} \stackrel{e^{\wedge}}{\rightarrow} K^{\wedge} \rightarrow 0
$$

Assume inductively that $f^{(j)}: M^{(j)} \rightarrow N$ has already been constructed for some $j<n / 2-1$. By the lemma, there exists a surjection

$$
\left(\begin{array}{l}
c \\
e
\end{array}\right): F=Z[\pi]^{m} \rightarrow K_{j}\left(M^{(j)}\right)=P_{j} \oplus K_{j}(M)
$$

with kernel $Q=P_{j+1}$, a f.g. projective $Z[\pi]$-module, such that

$$
\left[P_{j+1}\right]=-\left[P_{j}\right]-\chi\left(K_{j}(M)\right)=(-)^{j+1}\left(\sum_{r<j+1}(-)^{r} \chi\left(K_{r}(M)\right)\right) \in \tilde{K}_{0}(Z[\pi]) .
$$

Let $f^{(j+1)}: M^{(j+1)} \rightarrow N$ be the normal map obtained from $f^{(j)}$ by surgery on $m$ disjoint framed embedded spheres $S^{j} \subset M^{(j)}$ representing the corresponding $m$ $Z[\pi]$-module generators of $K_{j}\left(M^{(j)}\right)$. The trace normal bordism is denoted by

$$
\left(g^{(j+1)} ; f^{(j)}, f^{(j+1)}\right):\left(W^{(j+1)} ; M^{(j)}, M^{(j+1)}\right) \rightarrow N \times(I ; 0,1),
$$

and is such that

$$
\begin{aligned}
W^{(j+1)} & =M^{(j)} \times I \cup \cup S^{j \times D^{n-j}} \cup D^{j+1} \times D^{n-j} \\
& =M^{(j+1)} \times I \cup \cup D^{j+1} \times{S^{n-j-1}} \cup D^{j+1} \times D^{n-j} \\
& \simeq M^{(j)} \cup \cup{s^{j}} \cup D^{j+1} \\
& \simeq M^{(j+1)} \cup \cup S^{n-j-1} \cup D^{n-j} .
\end{aligned}
$$

It follows that

$$
\begin{aligned}
K_{r}\left(W^{(j+1)}, M^{(j)}\right) & =\left(\begin{array}{ll}
F & \text { if } r=j+1, \\
0 & \text { if } r \neq j+1,
\end{array}\right. \\
K_{r}\left(W^{(j+1)}, M^{(j+1)}\right) & =\left(\begin{array}{ll}
F^{*} & \text { if } r=n-j, \\
0 & \text { if } r \neq n-j,
\end{array}\right.
\end{aligned}
$$

and hence that

$$
\begin{gathered}
K_{r}\left(W^{(j+1)}\right)=K_{r}\left(M^{(j)}\right) \quad \text { if } r \neq j, j+1, \\
K_{r}\left(W^{(j+1)}\right)=K_{r}\left(M^{(j+1)}\right) \text { if } r \neq n-j-1, n-j .
\end{gathered}
$$


The exact sequences

$$
\begin{aligned}
& 0 \rightarrow K_{j+1}\left(M^{(j)}\right) \quad \rightarrow \quad K_{j+1}\left(W^{(j+1)}\right) \quad \rightarrow \quad K_{j+1}\left(W^{(j+1)}, M^{(j)}\right) \\
& \rightarrow \quad K_{j}\left(M^{(j)}\right) \quad \rightarrow \quad K_{j}\left(W^{(j+1)}\right) \quad \rightarrow \quad 0, \\
& 0 \rightarrow K_{n-j}\left(M^{(j+1)}\right) \rightarrow K_{n-j}\left(W^{(j+1)}\right) \rightarrow K_{n-j}\left(W^{(j+1)}, M^{(j+1)}\right) \\
& \rightarrow K_{n-j-1}\left(M^{(j+1)}\right) \rightarrow K_{n-j-1}\left(W^{(j+1)}\right) \quad \rightarrow \quad 0
\end{aligned}
$$

are naturally identified with the exact sequences

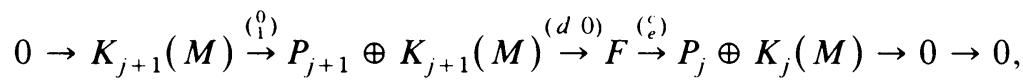

$$
\begin{aligned}
& 0 \rightarrow 0 \rightarrow P_{j}^{*} \stackrel{c^{*}}{\rightarrow} F^{*} \stackrel{d^{*}}{\rightarrow} P_{j+1}^{*} \stackrel{e^{\wedge}}{\rightarrow} K_{j}(M) \rightarrow 0
\end{aligned}
$$

respectively, with $d: P_{j+1} \rightarrow F$ the inclusion. In particular,

$$
\begin{aligned}
& K_{j+1}\left(M^{(j+1)}\right)=P_{j+1} \oplus K_{j+1}(M), \quad K_{j}\left(M^{(j+1)}\right)=0, \\
& K_{n-j-1}\left(M^{(j+1)}\right)=P_{j+1}^{*}, \quad K_{n-j}\left(M^{(j+1)}\right)=0,
\end{aligned}
$$

establishing the inductive step.

REMARK. The quadratic kernel of an $n$-dimensional normal map $f: M^{n} \rightarrow N^{n}$ with $\pi_{1}(N)=\pi$ is an $n$-dimensional quadratic Poincaré complex

$$
\left(C: C_{n} \stackrel{d}{\rightarrow} C_{n-1} \rightarrow \cdots \rightarrow C_{1} \stackrel{d}{\rightarrow} C_{0}, \psi\right)
$$

with $C$ a f.g. free $Z[\pi]$-module chain complex such that $H_{*}(C)=K_{*}(M)$ and $\psi$ a quadratic structure, such that the surgery obstruction of $f$ is determined by $(C, \psi)$

$$
\sigma_{*}(f)=(C, \psi) \in L_{n}(Z[\pi])
$$

(Ranicki [15]). Proposition 1.9 can also be proved by algebraic surgery on $(C, \psi)$.

B. The even-dimensional case. The quadratic kernel $(C, \psi)$ of a $2 i$-dimensional normal map $f: M^{2 i} \rightarrow N^{2 i}$ determines a (- $)^{i}$-quadratic form

$$
\left(K_{i}(M), \lambda: K_{i}(M) \times K_{i}(M) \rightarrow Z[\pi], \mu: K_{i}(M) \rightarrow Q_{(-)} i(Z[\pi])\right)
$$

which is nonsingular if all the kernel $Z[\pi]$-modules $H_{*}(C)=K_{*}(M)$ are f.g. projective. In the $(i-1)$-connected case $K_{r}(M)=0$ for $r \neq i, K_{i}(M)$ can be assumed to be f.g. free and $\left(K_{i}(M), \lambda, \mu\right)$ is the geometric intersection form defined by Wall [20].

For a $(2 i+1)$-dimensional normal map of manifolds with boundary

$$
(g, \partial g):\left(W^{2 i+1}, \partial W\right) \rightarrow\left(V^{2 i+1}, \partial V\right)
$$

the form $(\lambda, \mu)$ on $K_{i}(\partial W)$ restricts to $(0,0)$ on the submodule

$$
\operatorname{im}\left(\partial: K_{i+1}(W, \partial W) \rightarrow K_{i}(\partial W)\right) \subseteq K_{i}(\partial W) .
$$

If the kernel modules $K_{*}(\partial W), K_{*}(W)$ are all f.g. projective, then

$$
\operatorname{im}\left(K_{i+1}(W, \partial W) \rightarrow K_{i}(\partial W)\right)
$$


is a projective lagrangian of $\left(K_{i}(\partial W), \lambda, \mu\right)$. In particular, this is the case if $(g, \partial g)$ is $(i-1)$-connected, with $K_{r}(\partial W)=K_{r}(W)=0$ for $r \neq i$, when the lagrangian is in fact free.

THEOREM 1.11. Let $f: M^{2 i} \rightarrow N^{2 i}$ be a normal map of closed 2i-dimensional manifolds with $\pi_{1}(N)=\pi$ finite and $K_{*}(M) P L 1$ torsion, and such that for odd $i$ the Kervaire invariant $\sigma_{*}(f) \in L_{2 i}(Z[\{1\}])=Z_{2}$ is 0 . Then the surgery obstruction of $f$ is given by

$$
\begin{aligned}
\sigma_{*}(f) & =\partial\left((-)^{i} \sum_{j<i}(-)^{j} \chi\left(K_{j}(M)\right)\right) \\
& \in \operatorname{im}\left(\partial: \hat{H}_{2 i}\left(Z_{2}, \tilde{K}_{0}(Z[\pi])\right) \rightarrow L_{2 i}^{h}(Z[\pi])\right) .
\end{aligned}
$$

Proof. From Proposition 1.9 we have a normal bordant $(i-2)$-connected normal $\operatorname{map} f^{(i-1)}: M^{(i-1)} \rightarrow N$ such that

$$
K_{r}\left(M^{(i-1)}\right)=\left(\begin{array}{ll}
0 & \text { if } r<i-1 \text { or } r>i+1, \\
P_{i-1} \oplus K_{i-1}(M) & \text { if } r=i-1, \\
K_{i}(M) & \text { if } r=i, \\
P_{i-1}^{*} & \text { if } r=i+1,
\end{array}\right.
$$

with $P_{i-1}$ a f.g. projective $Z[\pi]$-module such that

$$
\left[P_{i-1}\right]=(-)^{i-1}\left(\sum_{j<i-1}(-)^{j} \chi\left(K_{j}(M)\right)\right) \in \tilde{K}_{0}(Z[\pi]) .
$$

By Lemma 1.10 there exists a surjection of a f.g. free $Z[\pi]$-module

$$
\left(\begin{array}{l}
c \\
e
\end{array}\right): F=Z[\pi]^{m} \rightarrow K_{i-1}\left(M^{(i-1)}\right)=P_{i-1} \oplus K_{i-1}(M),
$$

and $P_{i}=\operatorname{ker}\left(\left(\begin{array}{c}c \\ e\end{array}\right): F \rightarrow K_{i-1}\left(M^{(i-1)}\right)\right)$ is a f.g. projective $Z[\pi]$-module fitting into a short exact sequence

$$
0 \rightarrow P_{i} \stackrel{d}{\rightarrow} F \stackrel{(c)}{\rightarrow} P_{i-1} \oplus K_{i-1}(M) \rightarrow 0
$$

such that

$$
\left[P_{i}\right]=-\left[P_{i-1}\right]-\chi\left(K_{i-1}(M)\right)=(-)^{i}\left(\sum_{j<i}(-)^{j} \chi\left(K_{j}(M)\right)\right) \in \tilde{K}_{0}(Z[\pi]) .
$$

Let $f^{(i)}: M^{(i)} \rightarrow N$ be the $(i-1)$-connected normal map obtained from $f^{(i-1)}$ by surgery on the corresponding $m Z[\pi]$-module generators of $K_{i-1}\left(M^{(i-1)}\right)$. The trace normal bordism

$$
\left(g^{(i)} ; f^{(i-1)}, f^{(i)}\right):\left(W^{(i)} ; M^{(i-1)}, M^{(i)}\right) \rightarrow N \times(I ; 0,1)
$$

is such that

$$
\begin{aligned}
W^{(i)} & =M^{(i-1)} \times I \cup \cup S^{i-1} \times D^{i+1} \cup D^{i} \times D^{i+1} \\
& \simeq M^{(i)} \times I \cup \cup D^{i} \times S^{i} \cup D^{i} \times D^{i+1} \\
& \simeq M^{(i-1)} \cup \cup S^{i-1} \cup D^{i} \simeq M^{(i)} \cup \cup S^{i} \cup D^{i+1} .
\end{aligned}
$$


It follows that

and hence that

$$
\begin{gathered}
K_{r}\left(W^{(i)}, M^{(i-1)}\right)=\left(\begin{array}{ll}
F & \text { if } r=i \\
0 & \text { if } r \neq i
\end{array}\right. \\
K_{r}\left(W^{(i)}, M^{(i)}\right)=\left(\begin{array}{ll}
F^{*} & \text { if } r=i+1 \\
0 & \text { if } r \neq i+1
\end{array}\right.
\end{gathered}
$$

$$
\begin{array}{ll}
K_{r}\left(W^{(i)}\right)=K_{r}\left(M^{(i-1)}\right) & \text { if } r \neq i-1, i \\
K_{r}\left(W^{(i)}\right)=K_{r}\left(M^{(i)}\right) & \text { if } r \neq i, i+1 .
\end{array}
$$

The exact sequences

$$
\begin{aligned}
0 & \rightarrow K_{i}\left(M^{(i-1)}\right) \rightarrow K_{i}\left(W^{(i)}\right) \rightarrow K_{i}\left(W^{(i)}, M^{(i-1)}\right) \rightarrow K_{i-1}\left(M^{(i-1)}\right) \\
& \rightarrow K_{i-1}\left(W^{(i)}\right) \rightarrow 0, \\
0 & \rightarrow K_{i+1}\left(W^{(i)}, M^{(i-1)} \cup M^{(i)}\right) \rightarrow K_{i}\left(M^{(i-1)} \cup M^{(i)}, M^{(i-1)}\right) \quad\left(=K_{i}\left(M^{(i)}\right)\right) \\
& \rightarrow K_{i}\left(W^{(i)}, M^{(i-1)}\right) \rightarrow K_{i}\left(W^{(i)}, M^{(i-1)} \cup M^{(i)}\right) \rightarrow 0, \\
0 & \rightarrow K_{i+1}\left(M^{(i)}\right) \rightarrow K_{i+1}\left(W^{(i)}\right) \rightarrow K_{i+1}\left(W^{(i)}, M^{(i)}\right) \\
& \rightarrow K_{i}\left(M^{(i)}\right) \rightarrow K_{i}\left(W^{(i)}\right) \rightarrow 0
\end{aligned}
$$

are naturally identified with the exact sequences

$$
\begin{aligned}
& 0 \rightarrow K_{i}(M) \stackrel{\left(\begin{array}{l}
0 \\
1
\end{array}\right)}{\rightarrow} P_{i} \oplus K_{i}(M) \stackrel{(d) 0)}{\rightarrow} F \stackrel{\left(\begin{array}{c}
c \\
e
\end{array}\right)}{\rightarrow} P_{i-1} \oplus K_{i-1}(M) \rightarrow 0 \rightarrow 0, \\
& 0 \rightarrow P_{i}^{*} \stackrel{\left(\begin{array}{l}
0 \\
1
\end{array}\right)}{\rightarrow} P_{i} \oplus P_{i}^{*} \stackrel{(d 0)}{\rightarrow} F \stackrel{\left(\begin{array}{c}
c \\
e
\end{array}\right)}{\rightarrow} P_{i-1} \oplus K_{i-1}(M) \rightarrow 0, \\
& 0 \rightarrow 0 \rightarrow P_{i-1}^{*} \stackrel{c^{*}}{\rightarrow} F^{*} \stackrel{\left(\begin{array}{c}
0 \\
d^{*}
\end{array}\right)}{\rightarrow} P_{i} \oplus P_{i}^{*} \stackrel{\left(\begin{array}{cc}
1 & 0 \\
0 & e^{\wedge}
\end{array}\right)}{\rightarrow} P_{i} \oplus K_{i}(M) \rightarrow 0,
\end{aligned}
$$

respectively, and in particular

$$
K_{i-1}\left(M^{(i)}\right)=K_{i+1}\left(M^{(i)}\right)=0, \quad K_{i}\left(M^{(i)}\right)=P_{i} \oplus P_{i}^{*} .
$$

The $(-)^{i}$-quadratic forms $\left(K_{i}\left(M^{(i-1)}\right), \lambda^{(i-1)}, \mu^{(i-1)}\right),\left(K_{i}\left(M^{(i)}\right), \lambda^{(i)}, \mu^{(i)}\right)$ are such that $\left(\lambda^{(i-1)}, \mu^{(i-1)}\right) \oplus-\left(\lambda^{(i)}, \mu^{(i)}\right)$ restricts to $(0,0)$ on

$$
\begin{aligned}
\operatorname{im}\left(K_{i+1}\left(W^{(i)}, M^{(i-1)} \cup M^{(i)}\right)\right. & \left.\rightarrow K_{i}\left(M^{(i-1)} \cup M^{(i)}\right)\right) \\
= & \left\{\left(e^{\wedge}(x), 0, x\right) \mid x \in P_{i}^{*}\right\} \subset K_{i}(M) \oplus P_{i} \oplus P_{i}^{*}
\end{aligned}
$$

with $e^{\wedge}: P_{i}^{*} \rightarrow K_{i-1}(M)^{\wedge}=K_{i}(M)$ a surjection. Thus for all $x, y \in P_{i}^{*}$

$$
\begin{aligned}
& \lambda^{(i)}(x, y)=\lambda^{(i-1)}\left(e^{\wedge}(x), e^{\wedge}(y)\right) \in Z[\pi], \\
& \mu^{(i)}(x)=\mu^{(i-1)}\left(e^{\wedge}(x)\right) \in Q_{(-)} i(Z[\pi]) .
\end{aligned}
$$

Since $K_{i}\left(M^{(i-1)}\right)=K_{i}(M)$ is torsion,

$$
\left.\lambda^{(i-1)}\left(K_{i}(M) \times K_{i}(M)\right) \subseteq \text { (torsion subgroup of } Z[\pi]\right)=0 \subseteq Z[\pi]
$$

so that $\lambda^{(i-1)}=0$. Thus

$$
\lambda^{(i)}\left(P_{i}^{*} \times P_{i}^{*}\right)=\lambda^{(i-1)}\left(K_{i}(M) \times K_{i}(M)\right)=0
$$


and $P_{i}^{*}$ is a projective lagrangian of the nonsingular $(-)^{i}$-symmetric form $\left(K_{i}\left(M^{(i)}\right)\right.$, $\left.\lambda^{(i)}\right)$ on the f.g. free $Z[\pi]$-module $K_{i}\left(M^{(i)}\right)=P_{i} \oplus P_{i}^{*}$. For all $z \in K_{i-1}\left(M^{(i-1)}\right)$

$$
\lambda^{(i-1)}(z, z)=0=\mu^{(i-1)}(z)+(-)^{i} \overline{\mu^{(i-1)}(z)} \in Z[\pi]
$$

so that

$$
\begin{aligned}
\mu^{(i)}\left(P_{i}^{*}\right) & =\mu^{(i-1)}\left(K_{i}(M)\right) \\
& \subseteq \hat{H}_{i}\left(Z_{2} ; Z[\pi]\right)=\frac{\left\{a \in Z[\pi] \mid a+(-)^{i} \bar{a}=0\right\}}{\left\{b-(-)^{i} \bar{b} \mid b \in Z[\pi]\right\}} \subseteq Q_{(-)} i(Z[\pi]) .
\end{aligned}
$$

For $i$ even $\hat{H}_{i}\left(Z_{2}, Z[\pi]\right)=0$ (since $a \rightarrow \bar{a}$ is the oriented involution on $Z[\pi]$ ), so that $P_{i}^{*}$ is a projective lagrangian of the $(-)^{i}$-quadratic form $\left(K_{i}\left(M^{(i)}\right), \lambda^{(i)}, \mu^{(i)}\right)$. It is now immediate from Remark 1.4 that the surgery obstruction is given by

$$
\begin{aligned}
\sigma_{*}(f) & =\left(K_{i}\left(M^{(i)}\right), \lambda^{(i)}, \mu^{(i)}\right)=\partial\left(\left[P_{i}^{*}\right]\right) \\
& \in \operatorname{im}\left(\partial: \hat{H}_{2 i}\left(Z_{2} ; \tilde{K}_{0}(Z[\pi])\right) \rightarrow L_{2 i}^{h}(Z[\pi])\right)
\end{aligned}
$$

and Theorem 1.11 is proved in this case.

For $i$ odd $\hat{H}_{i}\left(Z_{2}, Z[\pi]\right)$ is a direct sum of copies of $Z_{2}$, one for each element in $\pi$ of order 1 or 2 . It remains to understand the failure of $\mu^{(i)}\left(P_{i}^{*}\right)$ to be 0 .

First consider the localization exact sequence

$$
\cdots \rightarrow L_{3}^{h, \text { tor }}(Z[\pi]) \rightarrow L_{2}^{h}(Z[\pi]) \rightarrow L_{2}^{h}(Q[\pi]) \rightarrow \cdots .
$$

Since $f: M \rightarrow N$ is a rational homotopy equivalence, the surgery obstruction $\sigma_{*}(f)=\left(K_{i}\left(M^{(i)}\right), \lambda^{(i)}, \mu^{(i)}\right) \in L_{2}^{h}(Z[\pi])$ has

$$
Q \otimes \sigma_{*}(f)=0 \in L_{2}^{h}(Q[\pi]) .
$$

(Alternatively, note that $Q \otimes P_{i}^{*}$ is a free lagrangian of $Q \otimes\left(K_{i}\left(M^{(i)}\right), \lambda^{(i)}, \mu^{(i)}\right)$.) Thus the surgery obstruction must come from the torsion $L$-group. Moreover, since we are dealing with a closed manifold surgery obstruction with finite fundamental group, the restriction to the Sylow-2-subgroup determines the surgery obstruction, and we may assume from now on that $\pi$ is a finite 2-group. But the image of $L_{3}^{h \text {.tor }}(Z[\pi])$ in $L_{2}^{h}(Z[\pi])$ is well understood in the oriented case of a finite 2-group. In particular, the image of $\sigma_{*}(f)$ in $L_{2}^{p}(Z[\pi])$ is either 0 or restricts to the simply-connected Kervaire problem (see e.g. Hambleton [8] or Taylor and Williams [18]). As we have assumed that the Kervaire invariant of $f$ is 0 , we thus have

$$
\sigma_{*}(f)=0 \in L_{2}^{p}(Z[\pi]) .
$$

To complete the proof, we proceed to analyze the $(-1)$-quadratic form $\left(K_{i}\left(M^{(i)}\right)\right.$, $\lambda^{(i)}, \mu^{(i)}$ ) with

$$
\left(K_{i}\left(M^{(i)}\right), \lambda^{(i)}\right)=\left(P_{i} \oplus P_{i}^{*},\left(\begin{array}{cc}
0 & 1 \\
-1 & 0
\end{array}\right)\right) .
$$

In view of Remark 1.4 it suffices to prove that the $(-1)$-quadratic form defined on the f.g. free $Z[\pi]$-module $V=P_{i} \oplus P_{i}^{*} \oplus P_{i} \oplus P_{i}^{*}$ by

$$
(V, \phi, \psi)=\left(K_{i}\left(M^{(i)}\right), \lambda^{(i)}, \mu^{(i)}\right) \oplus\left(P_{i} \oplus P_{i}^{*},\left(\begin{array}{cc}
0 & 1 \\
-1 & 0
\end{array}\right),\left(\begin{array}{ll}
0 & 1 \\
0 & 0
\end{array}\right)\right)
$$


is such that $\left(V, \phi^{\prime}, \psi\right)=0 \in L_{2}^{h}(Z[\pi])$. Now $F=0 \oplus P_{i}^{*} \oplus P_{i} \oplus 0$ is a free lagrangian of the $(-1)$-symmetric form $(V, \phi)$. Also,

$$
(V, \phi, \psi)=\sigma_{*}(f)=0 \in L_{2}^{p}(Z[\pi]),
$$

so $(V, \phi, \psi)$ admits a projective lagrangian $L$.

LeMMA 1.12. Let $(V, \phi, \psi)$ be a nonsingular (-1)-quadratic form over $Z(\pi), \pi a$ finite 2-group, such that $(V, \phi)$ admits a free lagrangian $F$ and $(V, \phi, \psi)$ admits a projective lagrangian $L$. Then $(V, \phi, \psi)$ admits a free lagrangian.

Proof. Since $\tilde{K}_{0}\left(\hat{Z}_{2}(\pi)\right)=0, \hat{L}=\hat{Z}_{2} \otimes_{Z} L$ is $\hat{Z}_{2}(\pi)$ free. Moreover, since $L$ is a lagrangian $V=L \oplus L^{*}$ so $\hat{Z}_{2} \otimes_{Z} V=\hat{L} \oplus \hat{L}^{*}$. Let $f_{1} \cdots f_{m}, f_{1}{ }^{*} \cdots f_{m}^{*}$ be a basis for $\hat{L}$ and $\hat{L}^{*}$, respectively, and suppose $e_{1} \cdots e_{m}, e_{1}^{*} \cdots e_{m}^{*}$ are bases for $F$, and $F^{*}$, respectively. Now, project $p: \hat{L} \rightarrow \hat{F}$, where $p$ is projection rel $\hat{F}^{*}$. We claim it is possible to choose $\hat{L}$ so that $p$ is an isomorphism. Indeed, the Jacobson radical $J$ in $\hat{Z}_{2}(\pi)$ satisfies $\hat{Z}_{2}(\pi) / J=F_{2}$, the field with two elements and tensoring over $\hat{Z}_{2}(\pi)$ with $F_{2}$ we obtain $\bar{p}: F_{2}^{m}\left(=F_{2} \otimes \hat{L}\right) \rightarrow F_{2}^{m}$. If $\bar{p}\left(\bar{f}_{1}\right), \ldots, \bar{p}\left(\bar{f}_{r}\right)$ are independent, but if $\bar{p}\left(\bar{f}_{r+1}\right)$ is dependent on the previous $r$ vectors, then $\bar{p}\left(\bar{f}_{r+1}^{*}\right)$ must be independent of $\bar{p}\left(\bar{f}_{1}\right), \ldots, \bar{p}\left(\bar{f}_{r}\right)$. Hence, after a finite number of interchanges, the claim is true over $F_{2}$. But this implies the truth of the claim over $\hat{Z}_{2}(\pi)$ by standard arguments. Hence there is an $\alpha \in \mathrm{GL}_{m}\left(\hat{Z}_{2}(\pi)\right)$ so that $p \cdot \alpha=I$, i.e.

$$
p\left(\alpha\left(f_{i}\right)\right)=e_{i}, \quad 1 \leqslant i \leqslant m .
$$

The element

$$
\left(\begin{array}{cc}
\alpha & 0 \\
0 & \left(\bar{\alpha}^{*}\right)^{-1}
\end{array}\right) \in \mathrm{Sp}(2 m)
$$

and it is possible to choose $f_{1}, \ldots, f_{m}$ such that $p\left(f_{i}\right)=e_{i}, 1 \leqslant i \leqslant m$. But then

$$
f_{i}=e_{i}+\sum \theta_{i j} e_{j}^{*}
$$

with the $\theta_{i j} \in \hat{Z}_{2}(\pi)$. Approximating these $\theta_{i j}(\bmod 2)$ by elements in $Z(\pi)$ which we denote $\tilde{\theta}_{i j}$, we have that

$$
\tilde{f}_{i}=e_{i}+\sum \tilde{\theta}_{i j} e_{j}
$$

form the basis of a free lagrangian of $(V, \phi, \psi)$. This completes the proof of the lemma, and hence also the theorem.

The following example illustrates the necessity for the assumption that the Kervaire invariant be zero in Theorem 1.11.

EXAMPLE 1.13. Let $f^{(3)}: M^{(3)}=S^{3} \times S^{3} \rightarrow N=S^{6}$ be the 2-connected 6-dimensional normal map with Kervaire invariant

$$
\sigma_{*}\left(f^{(3)}\right)=1 \in L_{6}(Z)=Z_{2},
$$

so that

$$
\left(K_{3}\left(M^{(3)}\right), \lambda^{(3)}, \mu^{(3)}\right)=\left(Z \oplus Z,\left(\begin{array}{cc}
0 & 1 \\
-1 & 0
\end{array}\right),\left(\begin{array}{ll}
1 & 1 \\
0 & 1
\end{array}\right)\right)
$$


Let $f^{(2)}: M^{(2)} \rightarrow N$ be the normal bordant 1-connected normal map obtained from $f^{(3)}$ by surgery on $(2,0) \in K_{3}\left(M^{(3)}\right)=Z \oplus Z$, with trace

$$
\left(g^{(3)} ; f^{(2)}, f^{(3)}\right):\left(W^{(3)} ; M^{(2)}, M^{(3)}\right) \rightarrow N \times(I ; 0,1) .
$$

Then

$$
\left(K_{3}\left(M^{(2)}\right), \lambda^{(2)}, \mu^{(2)}\right)=\left(Z_{2}, 0,1\right), \quad K_{2}\left(M^{(2)}\right)=Z_{2}
$$

and $\left(\lambda^{(2)}, \mu^{(2)}\right) \oplus-\left(\lambda^{(3)}, \mu^{(3)}\right)$ restricts to $(0,0)$ on the image of

$$
\left(\begin{array}{c}
e^{\wedge} \\
0 \\
1
\end{array}\right): K_{4}\left(W^{(3)}, M^{(2)} \cup M^{(3)}\right)=Z \rightarrow K_{3}\left(M^{(2)} \cup M^{(3)}\right)=Z_{2} \oplus Z \oplus Z .
$$

In particular,

$$
\mu^{(3)}(0 \oplus Z)=\mu^{(2)}\left(Z_{2}\right)=1 \neq 0 \in Q_{-1}(Z)=Z_{2} .
$$

C. The odd-dimensional case. The quadratic kernel $(C, \psi)$ of a $(2 i+1)$-dimensional normal map $f: M^{2 i+1} \rightarrow N^{2 i+1}$ determines a $(-)^{i+1}$-quadratic linking form on the torsion submodule $T_{i}(M) \subseteq K_{i}(M)$

$$
\left(T_{i}(M), \lambda: T_{i}(M) \times T_{i}(M) \rightarrow Q[\pi] / Z[\pi], \mu: T_{i}(M) \rightarrow Q_{(-)^{i}}(Q[\pi] / Z[\pi])\right)
$$

which is nonsingular if the kernel modules $K_{*}(M)$ are PL 1 torsion, so that in particular $T_{i}(M)=K_{i}(M)$. In the $(i-1)$-connected case $K_{r}(M)=0$ for $r \neq i$, $K_{i}(M)=T_{i}(M)$ is FPL 1 torsion and $\left(K_{i}(M), \lambda, \mu\right)$ coincides with the geometric linking form of Wall [19].

For a $(2 i+2)$-dimensional normal map of manifolds with boundary $(g, \partial g)$ : $\left(W^{2 i+2}, \partial W\right) \rightarrow\left(V^{2 i+2}, \partial V\right)$ the linking form $(\lambda, \mu)$ on $T_{i}(\partial W)$ restricts to $(0,0)$ on the submodule $\operatorname{im}\left(T_{i+1}(W, \partial W) \rightarrow T_{i}(\partial W)\right) \subset T_{i}(\partial W)$. If the kernel modules $K_{*}(\partial W), K_{*}(W)$ are all PL 1 torsion, then the submodule

$$
\begin{aligned}
\operatorname{im}\left(T_{i+1}(W, \partial W) \rightarrow T_{i}(\partial W)\right) & =\operatorname{im}\left(K_{i+1}(W, \partial W) \rightarrow K_{i}(\partial W)\right) \\
& \subseteq T_{i}(\partial W)=K_{i}(\partial W)
\end{aligned}
$$

is a PL 1 torsion lagrangian of $\left(T_{i}(\partial W), \lambda, \mu\right)$. Such is the case if $(g, \partial g)$ is $(i-1)$-connected with $K_{r}(\partial W)=K_{r}(W)=0$ for $r \neq i$ and $K_{i}(\partial W)=T_{i}(\partial W)$, $K_{i}(W)=T_{i}(W)$ PL 1 torsion modules, when the lagrangian is in fact FPL 1 torsion.

A sublagrangian of a nonsingular $(-)^{i+1}$-quadratic linking from $(K, \lambda, \mu)$ on a PL 1 torsion $Z[\pi]$-module $K$ is a submodule $L \subset K$ such that

(i) $L$ and $K / L$ are PL 1 torsion modules,

(ii) $(\lambda, \mu)$ restricts to $(0,0)$ on $L$,

(iii) the $Z[\pi]$-module morphism $K \rightarrow L^{\wedge} ; x \rightarrow(y \rightarrow \lambda(x, y))$ is onto.

It follows that the $Z[\pi]$-module $L^{\perp}=\operatorname{ker}\left(K \rightarrow L^{\wedge}\right)$ is PL 1 torsion, as is $L^{\perp} / L$. A lagrangian is a sublagrangian $L$ such that $L^{\perp}=L$, or equivalently such that the sequence $0 \rightarrow L \rightarrow K \rightarrow L^{\wedge} \rightarrow 0$ is exact. For any sublagrangian $L$ there is induced a nonsingular $(-)^{i+1}$-quadratic linking form $\left(L^{\perp} / L,[\lambda],[\mu]\right)$ such that the submodule

$$
\Delta=\left\{(x,[x]) \mid x \in L^{\perp}\right\} \subset K \oplus\left(L^{\perp} / L\right)
$$


is a lagrangian of $(K, \lambda, \mu) \oplus\left(L^{\perp} / L,-[\lambda],-[\mu]\right)$ isomorphic to $L^{\perp}$. If $K$ is FPL 1 then

$$
\chi\left(L^{\perp}\right)=\chi(K)-\chi\left(L^{\wedge}\right)=-\chi\left(L^{\wedge}\right) \in \tilde{K}_{0}(Z[\pi]),
$$

so that in the Witt group of nonsingular $(-)^{i+1}$-quadratic linking forms on FPL 1 torsion modules

$$
(K, \lambda, \mu)=\left(L^{\perp} / L,[\lambda],[\mu]\right) \oplus H_{(-)^{\prime+1}}^{\text {tor }}(L) \in L_{2 i+2}^{h, \text { tor }}(Z[\pi]) .
$$

In particular, if $\pi$ is finite and $L$ is a lagrangian

$$
(K, \lambda, \mu)=\partial^{\text {tor }}(\chi(L)) \in \operatorname{im}\left(\partial^{\text {tor }}: \hat{H}_{2 i+1}\left(Z_{2} ; \tilde{K}_{0}(Z[\pi])\right) \rightarrow L_{2 i+2}^{h \text { tor }}(Z[\pi])\right)
$$

(cf. Remark 1.6).

THEOREM 1.14. The surgery obstruction $\sigma_{*}(f) \in L_{2 i+1}^{h}(Z(\pi))$ of a normal map $f$ : $M^{2 i+1} \rightarrow N^{2 i+1}$ of closed $(2 i+1)$-dimensional manifolds with $\pi_{1}(N)=\pi$ finite and $K_{*}(M)=T_{*}(M) P L 1$ torsion is the image of

$$
\left(K_{i}(M), \lambda, \mu\right) \oplus H_{(-)^{\prime+1}}^{\text {tor }}(L) \in L_{2 i+2}^{h, \text { tor }}(Z[\pi]),
$$

with $L$ a PL 1 torsion $Z[\pi]$-module such that

$$
\chi(L)=(-)^{i}\left(\sum_{j<i}(-)^{j} \chi\left(K_{j}(M)\right)\right) \in \tilde{K}_{0}(Z[\pi]) .
$$

Corollary 1.15. If also $K_{i}(M)=0$, then

$$
\begin{aligned}
\sigma_{*}(f) & =\partial\left((-)^{i} \sum_{j<i}(-)^{j} \chi\left(K_{j}(M)\right)\right) \\
& \in \operatorname{im}\left(\partial: \hat{H}_{2 i+1}\left(Z_{2}, \tilde{K}_{0}(Z[\pi])\right) \rightarrow L_{2 i+1}^{h}(Z[\pi])\right) .
\end{aligned}
$$

Proof. Immediate from the theorem and the factorization

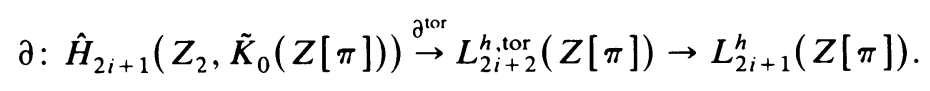

PROOF OF THE THEOREM. From Proposition 1.9 we have a normal bordant $(i-1)$-connected normal map $f^{(i)}: M^{(i)} \rightarrow N$ such that

$$
K_{r}\left(M^{(i)}\right)=\left(\begin{array}{ll}
0 & \text { if } r<i \text { or } r>i+1 \\
P_{i} \oplus K_{i}(M) & \text { if } r=i \\
P_{i}^{*} & \text { if } r=i+1
\end{array}\right.
$$

with $P_{i}$ a f.g. projective $Z(\pi)$-module such that

$$
\left[P_{i}\right]=(-)^{i}\left(\sum_{j<i}(-)^{j} \chi\left(K_{j}(M)\right)\right) \in \tilde{K}_{0}(Z[\pi]) .
$$

Choose an integral lattice $F=Z[\pi]^{m} \subset P_{i}$, so that there is defined a PL 1 torsion $Z[\pi]$-module $J=P_{i} / F$ with an exact sequence

$$
0 \rightarrow F \stackrel{d}{\rightarrow} P_{i} \stackrel{e}{\rightarrow} J \rightarrow 0
$$

and

$$
\chi(J)=\left[P_{i}\right] \in \tilde{K}_{0}(Z[\pi])
$$


Surgery on the corresponding $m$ elements of $P_{i} \subseteq K_{i}\left(M^{(i)}\right)$ results in a normal bordant $(i-1)$-connected normal map $f^{(i+1)}: M^{(i+1)} \rightarrow N$ with FPL 1 torsion kernel $K_{i}\left(M^{(i+1)}\right)$. The trace normal bordism

$$
\left(g^{(i+1)} ; f^{(i)}, f^{(i+1)}\right):\left(W^{(i+1)} ; M^{(i)}, M^{(i+1)}\right) \rightarrow N \times(I ; 0,1)
$$

is such that

$$
\begin{aligned}
W^{(i+1)} & =M^{(i)} \times I \cup \cup S^{i} \times D^{i+1} D^{i+1} \times D^{i+1} \\
& =M^{(i+1)} \times I \cup \cup D^{i+1} \times S^{i} \cup D^{i+1} \times D^{i+1} \\
& \simeq M^{(i)} \cup \cup S^{i} \cup D^{i+1} \simeq M^{(i+1)} \cup \cup S^{i} \cup D^{i+1} .
\end{aligned}
$$

It follows that

$$
\begin{aligned}
K_{r}\left(W^{(i+1)}, M^{(i)}\right) & =\left(\begin{array}{ll}
F & \text { if } r=i+1, \\
0 & \text { if } r \neq i+1,
\end{array}\right. \\
K_{r}\left(W^{(i+1)}, M^{(i+1)}\right) & =\left(\begin{array}{ll}
F^{*} & \text { if } r=i+1, \\
0 & \text { if } r \neq i+1
\end{array}\right.
\end{aligned}
$$

and hence that

$$
K_{r}\left(W^{(i+1)}\right)=K_{r}\left(M^{(i)}\right)=K_{r}\left(M^{(i)}\right) \quad \text { if } r \neq i, i+1 .
$$

The exact sequences

$$
\begin{aligned}
0 & \rightarrow K_{i+1}\left(M^{(i)}\right) \rightarrow K_{i+1}\left(W^{(i+1)}\right) \rightarrow K_{i+1}\left(W^{(i+1)}, M^{(i)}\right) \\
& \rightarrow K_{i}\left(M^{(i)}\right) \rightarrow K_{i}\left(W^{(i+1)}\right) \rightarrow 0 \\
0 & \rightarrow K_{i+1}\left(M^{(i+1)}\right) \rightarrow K_{i+1}\left(W^{(i+1)}\right) \rightarrow K_{i+1}\left(W^{(i+1)}, M^{(i+1)}\right) \\
& \rightarrow K_{i}\left(M^{(i+1)}\right) \rightarrow K_{i}\left(W^{(i+1)}\right) \rightarrow 0 \\
0 & \rightarrow K_{i+1}\left(M^{(i)} \cup M^{(i+1)}\right) \rightarrow K_{i+1}\left(W^{(i+1)}\right) \\
& \rightarrow K_{i+1}\left(W^{(i+1)}, M^{(i)} \cup M^{(i+1)}\right) \rightarrow K_{i}\left(M^{(i)} \cup M^{(i+1)}\right) \rightarrow K_{i}\left(W^{(i+1)}\right) \rightarrow 0
\end{aligned}
$$

are naturally identified with the exact sequences

$$
\begin{gathered}
0 \rightarrow P_{i}^{*} \stackrel{1}{\rightarrow} P_{i}^{*} \stackrel{0}{\rightarrow} F \stackrel{\left(\begin{array}{l}
d \\
0
\end{array}\right)}{\rightarrow} P_{i} \oplus K_{i}(M) \stackrel{\left(\begin{array}{ll}
e & 0 \\
0 & 1
\end{array}\right)}{\rightarrow} J \oplus K_{i}(M) \rightarrow 0, \\
0 \rightarrow 0 \rightarrow P_{i}^{*} \stackrel{d^{*}}{\rightarrow} F^{*} \stackrel{\left(\begin{array}{c}
h e^{\wedge} \\
0
\end{array}\right)}{\rightarrow} V \oplus K_{i}(M) \stackrel{\left(\begin{array}{ll}
k & 0 \\
0 & 1
\end{array}\right)}{\rightarrow} J \oplus K_{i}(M) \rightarrow 0, \\
0 \rightarrow P_{i}^{*} \stackrel{1}{\rightarrow} P_{i}^{*} \stackrel{0}{\rightarrow} P_{i} \oplus J^{\wedge} \oplus K_{i}(M) \stackrel{\left(\begin{array}{lll}
1 & 0 & 0 \\
0 & 0 & 1 \\
0 & h & 0 \\
0 & 0 & 1
\end{array}\right)}{\rightarrow} P_{i} \oplus K_{i}(M) \oplus V \oplus K_{i}(M) \\
\left(\begin{array}{cccc}
0 & 0 & k & 0 \\
0 & 1 & 0 & -1
\end{array}\right) \\
\rightarrow
\end{gathered}
$$


with $V$ a PL 1 torsion $Z[\pi]$-module fitting into an exact sequence $0 \rightarrow J^{\wedge} \stackrel{h}{\rightarrow} V \stackrel{k}{\rightarrow} J$ $\rightarrow 0$. The proof is completed by observing that the submodule

$$
\begin{aligned}
L & =\operatorname{im}\left(K_{i+1}\left(W^{(i+1)}, M^{(i)} \cup M^{(i+1)}\right) \rightarrow K_{i}\left(M^{(i)} \cup M^{(i+1)}, M^{(i)}\right)\right) \\
& =h\left(J^{\wedge}\right) \subset K_{i}\left(M^{(i+1)}\right)=V \oplus K_{i}(M)
\end{aligned}
$$

is a sublagrangian isomorphic to $J^{\wedge}$ of the nonsingular $(-)^{i+1}$-quadratic linking form

$$
\left(T_{i}\left(M^{(i+1)}\right)=K_{i}\left(M^{(i+1)}\right), \lambda^{(i+1)}, \mu^{(i+1)}\right)
$$

such that

$$
\left(L^{\perp} / L,\left[\lambda^{(i+1)}\right],\left[\mu^{(i+1)}\right]\right)=\left(K_{i}(M), \lambda, \mu\right),
$$

so that the surgery obstruction

$$
\sigma_{*}(f)=\sigma_{*}\left(f^{(i+1)}\right)=\sigma_{*}\left(f^{(i)}\right) \in L_{2 i+1}^{h}(Z[\pi])
$$

is the image of

$$
\left(T_{i}\left(M^{(i+1)}\right), \lambda^{(i+1)}, \mu^{(i+1)}\right)=\left(K_{i}(M), \lambda, \mu\right) \oplus H_{(-)^{i+1}}^{\text {tor }}(L) \in L_{2 i+2}^{h, \text { tor }}(Z[\pi]) .
$$

2. Algebraic surgery semi-invariants. In $\S 1$ we showed that the surgery obstruction $\sigma_{*}(f) \in L_{n}^{h}(Z[\pi])$ of an $n$-dimensional normal map $f: M^{n} \rightarrow N^{n}$ with $\pi_{1}(N)=\pi$ could in certain circumstances be expressed in terms of the projective semicharacteristic invariant

$$
\chi_{1 / 2}=\sum_{j<n / 2}(-)^{j} \chi\left(K_{j}(M)\right) \in \tilde{K}_{0}(Z[\pi]) .
$$

We shall now describe a general approach to such surgery semi-invariants using the algebraic theory of Ranicki [15], for any ring $A$ with involution ${ }^{-}: A \rightarrow A ; a \rightarrow \bar{a}$. (See Davis and Ranicki [22] for a further development of this approach.) We assume that the reader is already familiar with the definition of the quadratic $L$-groups $L_{*}(A)$ as the cobordism groups of quadratic Poincaré complexes over $A$. In dealing with quadratic Poincaré pairs $\left(f: C \rightarrow D,(\delta \psi, \psi) \in Q_{n}(f)\right)$ the terminology is contracted to $(D, C ; \delta \psi)$, and the algebraic mapping cone of $f$ is denoted $D / C$.

The projective $L$-group $L_{n}^{p}(A)$ is the cobordism group of $n$-dimensional quadratic Poincaré complexes $(C, \psi)$ over $A$, with $C$ an $n$-dimensional f.g. projective $A$-module chain complex

$$
C: C_{n} \stackrel{d}{\rightarrow} C_{n-1} \rightarrow \cdots \rightarrow C_{1} \stackrel{d}{\rightarrow} C_{0} .
$$

The finiteness obstruction of $C$ is the reduced projective class

$$
[C]=\sum_{r=0}^{n}(-)^{r}\left[C_{r}\right] \in \tilde{K}_{0}(A)
$$

and is such that $[C]=0$ if and only if $C$ is chain equivalent to an $n$-dimensional (stably) f.g. free $A$-module chain complex. The free $L$-group $L_{n}^{h}(A)$ is the cobordism group of $n$-dimensional quadratic Poincaré complexes $(C, \psi)$ over $A$ such that $C$ is 
an $n$-dimensional f.g. free $A$-module chain complex. The forgetful maps $L_{n}^{h}(A) \rightarrow$ $L_{n}^{p}(A) ;(C, \psi) \rightarrow(C, \psi)$ fit into an exact sequence

$$
\cdots \rightarrow L_{n}^{h}(A) \rightarrow L_{n}^{p}(A) \rightarrow L_{n}^{p, h}(A) \rightarrow L_{n-1}^{h}(A) \rightarrow \cdots
$$

with $L_{n}^{p, h}(A)$ the relative cobordism group of $n$-dimensional quadratic Poincaré pairs $(D, C ; \delta \psi)$ over $A$ such that $C$ is free and $D$ is projective. See Chapter 2 of Ranicki [16] for the definition of the relative cobordism group; in particular, $(D, C$; $\delta \psi)$ represents 0 in $L_{n}^{p, h}(A)$ if and only if it is the relative boundary of an $(n+1)$-dimensional quadratic Poincaré triad

$$
\left(\begin{array}{ccc}
C & \rightarrow & \delta C \\
\downarrow & \Gamma & \downarrow, \delta \delta \psi \\
D & \rightarrow & \delta D
\end{array}\right)
$$

with $\delta C$ free and $\delta D$ projective.

The $n$-dual of an $n$-dimensional f.g. projective $A$-module chain complex

$$
C: C_{n} \stackrel{d}{\rightarrow} C_{n-1} \rightarrow \cdots \rightarrow C_{1} \stackrel{d}{\rightarrow} C_{0}
$$

is the $n$-dimensional f.g. projective $A$-module chain complex

$$
C^{n-*}: C^{0} \stackrel{d^{*}}{\rightarrow} C^{1} \rightarrow \cdots \rightarrow C^{n-1} \stackrel{d^{*}}{\rightarrow} C^{n}
$$

with $C^{r}=C_{r}^{*}$. The $n$-dual has finiteness obstruction

$$
\left[C^{n-*}\right]=(-)^{n}[C]^{*} \in \tilde{K}_{0}(A) .
$$

If $(C, \psi)$ is an $n$-dimensional Poincaré complex over $A$, then $C^{n-*}$ is chain equivalent to $C$, so that

$$
[C]=\left[C^{n-*}\right]=(-)^{n}[C]^{*} \in \tilde{K}_{0}(A)
$$

representing an element $[C] \in \hat{H}_{n-1}\left(Z_{2}, \tilde{K}_{0}(A)\right)$. Similarly, for an $n$-dimensional quadratic Poincaré pair $(D, C ; \delta \psi) D / C$ is chain equivalent to $D^{n-*}$ so that

$$
[D]-[C]=(-)^{n}[D]^{*} \in \tilde{K}_{0}(A),
$$

and for an $(n+1)$-dimensional quadratic Poincaré triad $(\Gamma, \delta \psi)$ (as above) $\delta D /\left(D \cup_{C} \delta C\right)$ is chain equivalent to $\delta D^{n+1-*}$ so that

$$
[\delta D]-[D]-[\delta C]+[C]=(-)^{(n+1)}[\delta D]^{*} \in \tilde{K}_{0}(A) .
$$

THEOREM 2.1. The finiteness obstruction defines isomorphisms

$$
\chi: L_{n}^{p, h}(A) \stackrel{\sim}{\rightarrow} \hat{H}_{n-1}\left(Z_{2} ; \tilde{K}_{0}(A)\right) ;(D, C ; \delta \psi) \rightarrow[D] .
$$

Proof. Apply the 5-lemma to the map of exact sequences

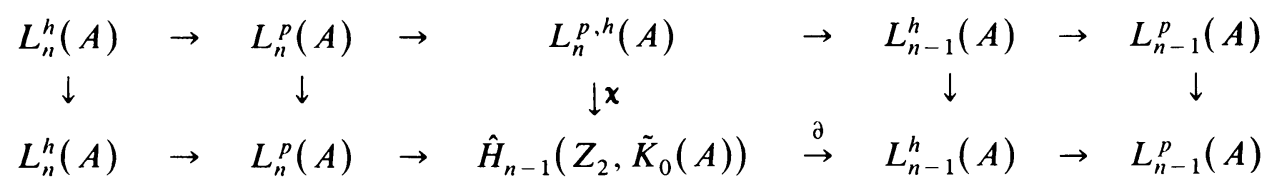


where the bottom sequence (of which (1.1) is the special case $A=Z[\pi]$ ) was obtained and proved exact in Ranicki [13].

REMARK 2.2. It follows from Theorem 2.1 that there are defined isomorphisms

$$
\operatorname{ker}\left(L_{n}^{h}(A) \rightarrow L_{n}^{p}(A)\right) \stackrel{\sim}{\rightarrow} \operatorname{coker}\left(L_{n+1}^{p}(A) \rightarrow \hat{H}_{n}\left(Z_{2}, \tilde{K}_{0}(A)\right)\right) ;
$$

$$
(C, \psi) \rightarrow[D]
$$

sending a projectively null-cobordant free $n$-dimensional quadratic Poincaré complex $(C, \psi)$ to the finiteness obstruction $[D]$ of any projective null-cobordism $(D, C$; $\delta \psi$ ). Remark 1.4 is an expression of this for forms and lagrangians, which is just the $(i-1)$-connected case for $n=2 i$.

Corollary 2.3. Let $(C, \psi)$ be an n-dimensional quadratic Poincaré complex over $A$ such that the homology $A$-modules $H_{r}(C)(0 \leqslant r \leqslant n)$ are f.g. projective, and such that $H_{i}(C)=0$ if $n=2 i$. Then $(C, \psi)=0 \in L_{n}^{p}(A)$, and if $C$ is free then $(C, \psi) \in$ $L_{n}^{h}(A)$ is given by

$$
(C, \psi)=\partial\left(\chi_{1 / 2}(C)\right) \in \operatorname{im}\left(\partial: \hat{H}_{n}\left(Z_{2}, \tilde{K}_{0}(A)\right) \rightarrow L_{n}^{h}(A)\right),
$$

where the semicharacteristic is defined by

$$
\chi_{1 / 2}(C)=\sum_{r=0}^{i}(-)^{r}\left[H_{r}(C)\right] \in \tilde{K}_{0}(A) \quad(n=2 i \text { or } 2 i+1) .
$$

Proof. $C$ is chain equivalent to the chain complex of homology modules

$$
H(C): H_{n}(C) \stackrel{0}{\rightarrow} H_{n-1}(C) \rightarrow \cdots \rightarrow H_{1}(C) \stackrel{0}{\rightarrow} H_{0}(C),
$$

so that there is defined a projective null-cobordism $(D, C ; \delta \psi)$ of $(C, \psi)$ with

$$
D: H_{n}(C) \stackrel{0}{\rightarrow} H_{n-1}(C) \rightarrow \cdots \rightarrow H_{i+1}(C) \rightarrow 0 \rightarrow \cdots \rightarrow 0
$$

such that

$$
[D]=\sum_{r=i+1}^{n}(-)^{r}\left[H_{r}(C)\right]=[C]-\chi_{1 / 2}(C) \in \tilde{K}_{0}(A) .
$$

If $[C]=0 \in \tilde{K}_{0}(A)$, then

$$
[D]=-\chi_{1 / 2}(C)=\chi_{1 / 2}(C) \in \hat{H}_{n}\left(Z_{2}, \tilde{K}_{0}(A)\right) .
$$

Now apply Remark 2.2 .

Remark 2.4. If $A$ is a semisimple ring with involution, then every projective $(2 i+1)$-dimensional quadratic Poincare complex $(C, \psi)$ over $A$ is such that the homology $A$-modules $H_{r}(C)(0 \leqslant r \leqslant 2 i+1)$ are f.g. projective, so that $L_{2 i+1}^{p}(A)=$ 0 (cf. Ranicki [14]) and the semicharacteristic defines an isomorphism

$$
\begin{gathered}
\chi_{1 / 2}: L_{2 i+1}^{h}(A) \stackrel{\sim}{\rightarrow} \operatorname{coker}\left(L_{2 i+2}^{p}(A) \rightarrow \hat{H}_{2 i+1}\left(Z_{2}, \tilde{K}_{0}(A)\right)\right) ; \\
(C, \psi) \rightarrow \chi_{1 / 2}(C) .
\end{gathered}
$$

(See Davis [7] for a recent account of the applications of the semicharacteristic in surgery theory.) 
More generally, given any *-invariant subgroup $X \subseteq \tilde{K}_{0}(A)$, there are defined the intermediate quadratic $L$-groups $L_{n}^{X}(A)$ of $n$-dimensional quadratic Poincaré complexes $(C, \psi)$ over $A$ with $C$ projective and $[C] \in X \subseteq \tilde{K}_{0}(A)$. Given $*$-invariant subgroups $Y \subseteq X \subseteq \tilde{K}_{0}(A)$, there are defined relative cobordism groups $L_{n}^{X, Y}(A)$ of $n$-dimensional quadratic Poincaré pairs $(D, C ; \delta \psi)$ over $A$ with $[C] \in Y,[D] \in X \subseteq$ $\tilde{K}_{0}(A)$, fitting into an exact sequence

$$
\cdots \rightarrow L_{n}^{Y}(A) \rightarrow L_{n}^{X}(A) \rightarrow L_{n}^{X, Y}(A) \rightarrow L_{n-1}^{Y}(A) \rightarrow \cdots .
$$

The finiteness obstruction defines isomorphisms

$$
\chi: L_{n}^{X, Y}(A) \stackrel{\sim}{\rightarrow} \hat{H}_{n-1}\left(Z_{2} ; Y / X\right) ;(D, C ; \delta \psi) \rightarrow[D] .
$$

The isomorphisms of Theorem 2.1 are just the special case $Y=\{0\} \subseteq X=\tilde{K}_{0}(A)$, since

$$
L_{*}^{\{0\} \subseteq \tilde{K}_{0}(A)}(A)=L_{*}^{h}(A), \quad L_{*}^{\tilde{K}_{0}(A)}(A)=L_{*}^{p}(A) .
$$

Let $Z_{2}$ act on the Whitehead torsion group $\tilde{K}_{1}(A)$ by

$$
*: \tilde{K}_{1}(A) \rightarrow \tilde{K}_{1}(A) ; \tau\left(a: A^{m} \rightarrow A^{m}\right) \rightarrow \tau\left(a^{*}: A^{m} \rightarrow A^{m}\right),
$$

where $a^{*}=\left(\bar{a}_{j i}\right)$ if $a=\left(a_{i j}\right)$. Given a $*$-invariant subgroup $X \subseteq \tilde{K}_{1}(A)$ there are defined the intermediate $L$-groups $L_{n}^{X}(A)$ of $n$-dimensional quadratic Poincaré complexes $(C, \psi)$ over $A$ with $C$ a based f.g. free $A$-module chain complex and the Poincaré duality chain equivalence $(1+T) \psi_{0}: C^{n-*} \stackrel{\sim}{\rightarrow} C$ such that

$$
\tau\left((1+T) \psi_{0}: C^{n-*} \stackrel{\sim}{\rightarrow} C\right) \in X \subseteq \tilde{K}_{1}(A) .
$$

In particular,

$$
L_{*}^{\{0\} \subseteq \tilde{K}_{1}(A)}(A)=L_{*}^{s}(A), \quad L_{*}^{\tilde{K}_{1}(A)}(A)=L_{*}^{h}(A) .
$$

Given *-invariant subgroups $Y \subseteq X \subseteq \tilde{K}_{1}(A)$ there are defined relative cobordism groups $L_{n}^{X, Y}(A)$ of $n$-dimensional quadratic Poincaré pairs $(D, C ; \delta \psi)$ over $A$ with $D, C$ based f.g. free and

$$
\begin{gathered}
\tau(C, \psi) \equiv \tau \cdot\left((1+T) \psi_{0}: C^{n-1-*} \rightarrow C\right) \in Y \subseteq \tilde{K}_{1}(A), \\
\tau(D, C ; \delta \psi) \equiv \tau\left((1+T) \delta \psi_{0}: D^{n-*} \rightarrow D / C\right) \in X \subseteq \tilde{K}_{1}(A) .
\end{gathered}
$$

The Whitehead torsion defines isomorphisms

$$
\tau: L_{n}^{X, Y}(A) \stackrel{\sim}{\rightarrow} \hat{H}_{n-1}\left(Z_{2} ; X / Y\right) ;(D, C ; \delta \psi) \rightarrow \tau(D, C ; \delta \psi),
$$

by analogy with Theorem 2.1 .

Let now $S \subset A$ be a multiplicative subset of central nonzero divisors invariant under the involution, so that the localization

$$
S^{-1} A=\{a / s \mid a \in A, s \in S\}
$$

is defined and the inclusion $A \rightarrow S^{-1} A ; a \rightarrow a / 1$ is a morphism of rings with involution. An $A$-module $M$ is $S$-torsion if $S^{-1} M=0$, or equivalently if for all $x \in M$ there exists $s \in S$ such that $s x=0 \in M$. Let $K_{1}(A, S)$ denote the Grothendieck group of stable isomorphism classes $\chi^{S}(M)$ of PL $1 S$-torsion 
$A$-modules $M$, i.e. $A$-modules with a f.g. projective resolution $0 \rightarrow P_{1} \stackrel{d}{\rightarrow} P_{0} \stackrel{e}{\rightarrow} M \rightarrow 0$ such that $S^{-1} d \in \operatorname{Hom}_{S^{-1} A}\left(S^{-1} P_{1}, S^{-1} P_{0}\right)$ is an isomorphism, subject to the relations

$$
\chi^{S}(M)-\chi^{S}\left(M^{\prime}\right)+\chi^{S}\left(M^{\prime \prime}\right)=0 \in K_{1}(A, S)
$$

for exact sequences $0 \rightarrow M \rightarrow M^{\prime} \rightarrow M^{\prime \prime} \rightarrow 0$. The $S$-torsion duality defines an involution

$$
\text { *: } K_{1}(A, S) \rightarrow K_{1}(A, S) ; \chi^{S}(M) \rightarrow \chi^{S}\left(M^{\wedge}\right)
$$

with $A$ acting on $M^{\wedge}=\operatorname{Hom}_{A}\left(M, S^{-1} A / A\right)$ by

$$
A \times M^{\wedge} \rightarrow M^{\wedge} ;(a, \phi) \rightarrow(x \rightarrow \phi(x) \bar{a}),
$$

so that $M^{\wedge}$ has the dual f.g. projective resolution

$$
0 \rightarrow P_{0}^{*} \stackrel{d^{*}}{\rightarrow} P_{1}^{*} \stackrel{e^{\wedge}}{\rightarrow} M^{\wedge} \rightarrow 0
$$

with

$$
\begin{aligned}
e^{\wedge}: P_{1}^{*} \rightarrow M^{\wedge} ; \theta \rightarrow(e(x) \rightarrow \theta(y) / s) & \\
& \left(x \in P_{0}, y \in P_{1}, s \in S, s x=d(y) \in P_{0}\right) .
\end{aligned}
$$

The localization exact sequence of algebraic $K$-theory

$$
\tilde{K}_{1}(A) \rightarrow \tilde{K}_{1}\left(S^{-1} A\right) \stackrel{j}{\rightarrow} K_{1}(A, S) \stackrel{\partial}{\rightarrow} \tilde{K}_{0}(A) \rightarrow \tilde{K}_{0}\left(S^{-1} A\right)
$$

involves the maps induced in $\tilde{K}_{0}$ and $\tilde{K}_{1}$ by the inclusion $A \rightarrow S^{-1} A$, and also the maps

$$
\begin{aligned}
& j: \tilde{K}_{1}\left(S^{-1} A\right) \rightarrow K_{1}(A, S) ; \\
& \tau\left(a / s: S^{-1} A^{m} \rightarrow S^{-1} A^{m}\right) \rightarrow \chi^{S}\left(A^{m} / a\left(A^{m}\right)\right)-\chi^{S}\left(A^{m} / s A^{m}\right), \\
& \text { ə: } K_{1}(A, S) \rightarrow \tilde{K}_{0}(A) ; \chi^{S}(M) \rightarrow \chi(M)=\left[P_{0}\right]-\left[P_{1}\right],
\end{aligned}
$$

which are such that $j^{*}={ }^{*} j, \partial^{*}=-*^{*}$.

An $A$-module chain complex $C$ is $S^{-1} A$-acyclic if $S^{-1} C=S^{-1} A \otimes_{A} C$ is an acyclic $S^{-1} A$-module chain complex $\left(H_{*}\left(S^{-1} C\right)=S^{-1} H_{*}(C)=0\right)$, or equivalently if the homology $A$-modules $H_{*}(C)$ are $S$-torsion. An $S^{-1} A$-acyclic $n$-dimensional f.g. projective $A$-module chain complex $C$ has an $S$-torsion characteristic $\chi^{S}(C) \in$ $K_{1}(A, S)$ such that

$$
\partial\left(\chi^{S}\left(C^{n-*}\right)\right)=(-)^{n-1} \chi^{S}(C)^{*} \in K_{1}(A, S) .
$$

If $C$ is such that each $H_{r}(C)(0 \leqslant r \leqslant n-1)$ is a PL $1 S$-torsion $A$-module, then

$$
\chi^{S}(C)=\sum_{r=0}^{n-1}(-)^{r} \chi^{S}\left(H_{r}(C)\right) \in K_{1}(A, S) .
$$

The localization exact sequence of algebraic $L$-theory for a $*$-invariant subgroup $X \subseteq K_{1}(A, S)$

$$
\cdots \rightarrow L_{n}^{\partial(X)}(A) \rightarrow L_{n}^{j^{1}(X)}\left(S^{-1} A\right) \rightarrow L_{n}^{X}(A, S) \rightarrow L_{n-1}^{\partial(X)}(A) \rightarrow \cdots
$$


involves the cobordism groups $L_{n}^{X}(A, S)$ of $S^{-1} A$-acyclic $(n-1)$-dimensional quadratic Poincaré complexes $(C, \psi)$ over $A$ with $\chi^{S}(C) \in X \subseteq K_{1}(A, S)$. If $X$ contains the elements $\chi^{S}(A / s A)(s \in S)$ the $S$-torsion $L$-groups $L_{2 i}^{X}(A, S)$ (resp. $\left.L_{2 i+1}^{X}(A, S)\right)$ may be identified with the Witt groups of $(-)^{i}$-quadratic linking forms (resp. formations) defined using PL $1 S$-torsion $A$-modules $M$ with $\chi^{S}(M) \in X \subseteq$ $K_{1}(A, S)$, otherwise they are the Witt groups of formal differences of such objects with virtual $S$-torsion characteristic in $X$. See Chapter 3 of Ranicki [16] for the details, and also Pardon [11], Carlsson-Milgram [4] for the localization exact sequence of Witt groups.

THEOREM 2.5. The algebraic L-theory localization exact sequences for $*$-invariant subgroups $Y \subseteq X \subseteq K_{1}(A, S)$ fit together in a commutative diagram of exact sequences

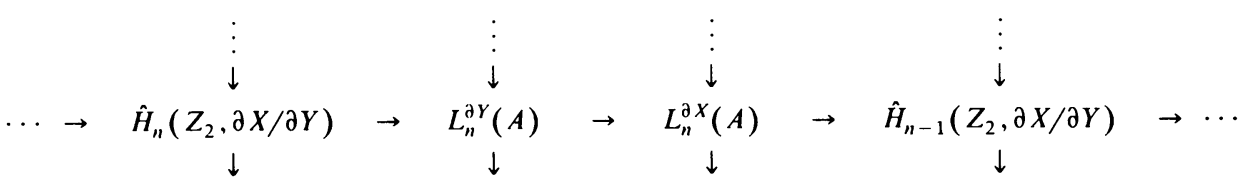

$$
\begin{aligned}
& \cdots \rightarrow \hat{H}_{n}\left(Z_{2}, j^{-1} X / j^{-1} Y\right) \rightarrow L_{n}^{j^{-1} Y}\left(S^{-1} A\right) \rightarrow L_{n}^{j^{-1} X}\left(S^{-1} A\right) \rightarrow \hat{H}_{n-1}\left(Z_{2}, j^{-1} X / j^{-1} Y\right) \rightarrow \cdots \\
& \cdots \rightarrow \hat{H}_{n}\left(Z_{2}, X / Y\right) \rightarrow L_{n}^{Y}(A, S) \rightarrow L_{n}^{X}(A, S) \rightarrow \hat{H}_{n-1}\left(Z_{2}, X / Y\right) \rightarrow \cdots
\end{aligned}
$$

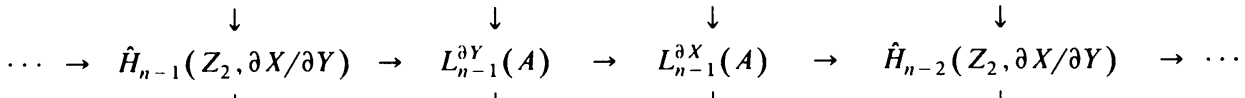

Proof. This is just a restatement of Proposition 3.7.1 of Ranicki [16].

\section{Define}

$$
\begin{aligned}
& \tilde{K}_{0}(A)^{S}=\operatorname{ker}\left(\tilde{K}_{0}(A) \rightarrow \tilde{K}_{0}\left(S^{-1} A\right)\right)=\operatorname{im}\left(\partial: K_{1}(A, S) \rightarrow \tilde{K}_{0}(A)\right), \\
& L_{n}^{h}(A, S)=L_{n}^{\operatorname{ker}\left(\partial: K_{1}(A, S) \rightarrow \tilde{K}_{0}(A)\right)}(A, S), \\
& L_{n}^{p}(A, S)=L_{n}^{K_{1}(A, S)}(A, S) .
\end{aligned}
$$

In the case

$$
Y=\operatorname{ker}\left(\partial: K_{1}(A, S) \rightarrow \tilde{K}_{0}(A)\right) \subseteq X=K_{1}(A, S),
$$

the diagram of Theorem 2.5 collapses to the commutative braid of exact sequences

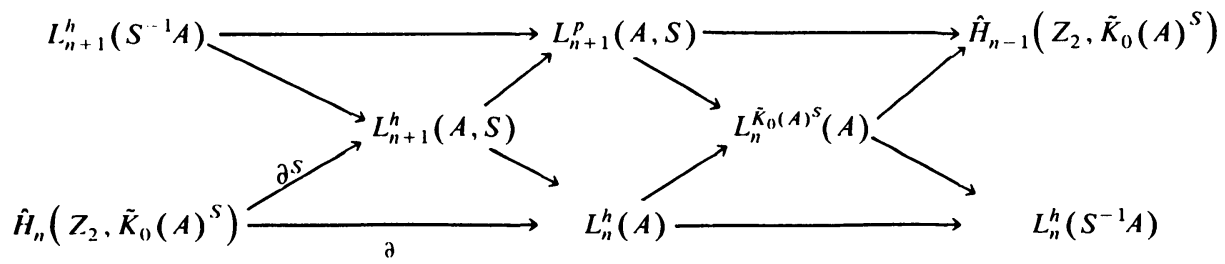


In particular, for $(A, S)=(Z[\pi], Z-\{0\})(\pi$ finite $) \tilde{K}_{0}(A)^{S}=\tilde{K}_{0}(Z[\pi])$ by Swan's theorem, and this is just the braid (1.3) with

$$
L_{*}^{h}(A, S)=L_{*}^{h \cdot \operatorname{tor}}(Z[\pi]), \quad L_{*}^{p}(A, S)=L_{*}^{p, \text { tor }}(Z[\pi]) .
$$

Given *-invariant subgroups $Y \subseteq X \subseteq K_{1}(A, S)$ there are defined relative cobordism groups $L_{n}^{X, Y}(A, S)$ of $S^{-1} A$-acyclic $(n-1)$-dimensional quadratic Poincaré pairs $(D, C ; \delta \psi)$ over $A$ such that

$$
\chi^{S}(C) \in Y, \quad \chi^{S}(D) \in X \subseteq K_{1}(A, S),
$$

fitting into an exact sequence

$$
\cdots \rightarrow L_{n}^{Y}(A, S) \rightarrow L_{n}^{X}(A, S) \rightarrow L_{n}^{X . Y}(A, S) \rightarrow L_{n-1}^{Y}(A, S) \rightarrow \cdots .
$$

THEOREM 2.6. The S-torsion characteristic defines isomorphisms

$$
\chi^{S}: L_{n}^{X, Y}(A, S) \stackrel{\sim}{\rightarrow} \hat{H}_{n-1}\left(Z_{2}, X / Y\right) ; \quad(D, C ; \delta \psi) \rightarrow \chi^{S}(D) .
$$

Proof. By analogy with Theorem 2.1.

REMARK 2.7. By analogy with the isomorphisms of Remark 2.2 the $S$-torsion characteristic also defines isomorphisms

$$
\operatorname{ker}\left(L_{n}^{Y}(A, S) \rightarrow L_{n}^{X}(A, S)\right) \stackrel{\sim}{\rightarrow} \operatorname{coker}\left(L_{n+1}^{X}(A, S) \rightarrow \hat{H}_{n}\left(Z_{2}, X / Y\right)\right) ;
$$

$$
(C, \psi) \rightarrow \chi^{S}(D),
$$

with $(D, C ; \delta \psi)$ any $S^{-1} A$-acyclic null-cobordism of $(C, \psi)$ such that $\chi^{S}(D) \in X \subseteq$ $K_{1}(A, S)$. Remark 1.6 is an expression of the isomorphism

$$
\begin{aligned}
\operatorname{ker}\left(L_{2 i}^{h}(A, S) \rightarrow L_{2 i}^{p}(A, S)\right) & \stackrel{\sim}{\rightarrow} \operatorname{coker}\left(L_{2 i+1}^{h}(A, S) \rightarrow \hat{H}_{2 i}\left(Z_{2}, \tilde{K}_{0}(A)^{S}\right)\right) ; \\
& (C, \psi) \rightarrow \chi(D)
\end{aligned}
$$

in terms of linking forms and torsion lagrangians, which is just the $(i-2)$-connected case.

Corollary 2.3 has only a partial analogue for the torsion $L$-groups:

Corollary 2.8. Let $(C, \psi)$ be an n-dimensional quadratic Poincaré complex over $A$ such that the homology $A$-modules $H_{*}(C)$ are PL $1 S$-torsion, such that $H_{i-1}(C)=$ $H_{i}(C)=0$ if $n=2 i$ and $H_{i}(C)=0$ if $n=2 i+1$. Then $(C, \psi)=0 \in L_{n+1}^{p}(A, S)$, and if $C$ is free

$$
(C, \psi)=\partial s\left(\sum_{r=0}^{i-1}(-)^{r} \chi\left(H_{r}(C)\right)\right) \in \operatorname{im}\left(\partial^{s}: \hat{H}_{n}\left(Z_{2} ; \tilde{K}_{0}(A)^{S}\right) \rightarrow L_{n+1}^{h}(A, S)\right) .
$$

Proof. If $C$ is any $n$-dimensional f.g. projective $A$-module chain complex with $\mathrm{PL}$ $1 S$-torsion homology $A$-modules $H_{*}(C)$, then for any f.g. projective $A$-module resolutions

$$
0 \rightarrow Q_{r} \stackrel{d}{\rightarrow} P_{r} \rightarrow H_{r}(C) \rightarrow 0 \quad(0 \leqslant r \leqslant n-1)
$$


$C$ is chain equivalent to the chain complex

$$
C^{\prime}: Q_{n-1} \stackrel{\left(\begin{array}{l}
d \\
0
\end{array}\right)}{\rightarrow} P_{n-1} \oplus Q_{n-2} \stackrel{\left(\begin{array}{ll}
0 & d \\
0 & 0
\end{array}\right)}{\rightarrow} P_{n-2} \oplus Q_{n-3} \rightarrow \cdots \rightarrow P_{1} \oplus Q_{0} \stackrel{(0}{\rightarrow}{ }^{d)} P_{0} .
$$

Now if $(C, \psi)$ satisfies the stated conditions, there is defined an $S^{-1} A$-acyclic null-cobordism $(D, C ; \delta \psi)$ with

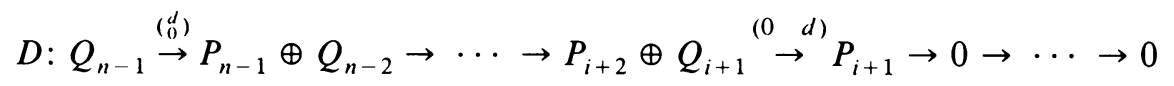

such that

$$
\chi^{S}(D)=\sum_{r=i+1}^{n}(-)^{r} \chi^{S}\left(H_{r}(C)\right) \in K_{1}(A, S) .
$$

Now apply Remark 2.7 .

A full analogue of Corollary 2.3 for the torsion $L$-groups would be that any $2 i$-dimensional quadratic Poincaré complex $(C, \psi)$ over $A$ with PL $1 S$-torsion homology $A$-modules $H_{*}(C)$ is such that

$$
\begin{aligned}
(C, \psi) & =\partial^{S}\left(\sum_{r=0}^{i-1}(-)^{r} \chi\left(H_{r}(C)\right)\right) \\
& \in \operatorname{im}\left(\partial^{S}: \hat{H}_{2 i}\left(Z_{2}, \tilde{K}_{0}(A)^{S}\right) \rightarrow L_{2 i+1}^{h}(A, S)\right) \\
& =\operatorname{ker}\left(L_{2 i+1}^{h}(A, S) \rightarrow L_{2 i+1}^{p}(A, S)\right) .
\end{aligned}
$$

However, Example 1.13 shows that this is false for $(A, S)=(Z, Z-\{0\})$ with $i$ odd, for in that case $(C, \psi)$ is an $S$-acyclic 2-dimensional quadratic Poincaré complex over $A$ with

$$
(C, \psi)=1 \in L_{2}(Z)=Z_{2}, \quad H_{0}(C)=H_{1}(C)=Z_{2}, \quad H_{2}(C)=0,
$$

so that $H_{*}(C)$ is PL $1 S$-torsion while $\tilde{K}_{0}(A)^{S}=0$. The full analogue does hold for the torsion $L$-groups if $(A, S)$ is such that $\hat{H}_{*}\left(Z_{2}, S^{-1} A / A\right)=0$, with $Z_{2}$ acting on $S^{-1} A / A$ by the involution. In that case there is defined an $S^{-1} A$-acyclic null-cobor$\operatorname{dism}(D, C ; \delta \psi)$ with

$$
D: Q_{2 i-1} \stackrel{\left(\begin{array}{l}
d \\
0
\end{array}\right)}{\rightarrow} P_{2 i-1} \oplus Q_{2 i-2} \rightarrow \cdots \rightarrow P_{i+1} \oplus Q_{i} \stackrel{(0 \stackrel{d}{\rightarrow})}{\rightarrow} P_{i} \rightarrow 0 \rightarrow \cdots \rightarrow 0
$$

such that

$$
\chi^{S}(D)=\sum_{r=i}^{2 i-1}(-)^{r} \chi\left(H_{r}(C)\right) \in K_{1}(A, S),
$$

and Remark 2.7 applies as before. For arbitrary $(A, S)$ the $i$ th quadratic linking Wu class of $(C, \psi)$ (defined in Chapter 3.3 of Ranicki [16])

$$
v_{S}^{i}(\psi): H^{i}(C)=H_{i}(C) \rightarrow \hat{H}_{1}\left(Z_{2}, S^{-1} A / A\right)
$$

is an obstruction to defining such a null-cobordism $(D, C ; \delta \psi)$. In Example 1.13 this obstruction is nonzero, with

$$
v_{S}^{1}(\psi)=1: H^{1}(C)=H_{1}(C)=Z_{2} \rightarrow \hat{H}_{1}\left(Z_{2}, Q / Z\right)=Z_{2} \quad(i=1)
$$


detected by the Kervaire invariant. If $(A, S)=(Z[\pi]$, odd $\})$ for a finite 2-group $\pi$, then $\hat{H}_{*}\left(Z_{2}, S^{-1} A / A\right)=0$ and also every f.g. odd torsion $Z[\pi]$-module is PL 1 (cf. Remark 1.5); it follows that the surgery obstruction of an $n$-dimensional normal map $f: M^{n} \rightarrow N^{n}$ with $\pi_{1}(M)=\pi$ a finite 2-group, $K_{*}(M)$ odd torsion and $K_{i}(M)=0$ if $n=2 i+1$ is given by

$$
\begin{array}{r}
\sigma_{*}(f)=\partial\left(\sum_{r=0}^{i-1}(-)^{r} \chi\left(K_{r}(M)\right)\right) \in \operatorname{im}\left(\partial: \hat{H}_{n}\left(Z_{2}, \tilde{K}_{0}(Z[\pi])\right) \rightarrow L_{n}^{h}(Z[\pi])\right) \\
(n=2 i \text { or } 2 i+1) .
\end{array}
$$

Note also that this example contradicts the quadratic even-dimensional case of Proposition 7.1 of Ranicki [15, I]: if $A$ is a Dedekind ring with involution and $(C, \psi)$ is a $2 i$-dimensional quadratic Poincaré complex over $A$ the cobordism class $(C, \psi) \in$ $L_{2 i}^{p}(A)$ is not in general the $i$-fold skew-suspension $\bar{S}^{i}\left(F^{i}(C), \psi\right)$ of the nonsingular $(-)^{i}$-quadratic form on the f.g. projective $A$-module $F^{i}(C)=H^{i}(C) /$ torsion. However, the instant surgery obstruction formula of Proposition 4.3 of $[15, \mathrm{I}]$ (for any ring with involution $A$ ) applies to show that the cobordism class $(C, \psi) \in L_{2 i}^{p}(A)$ is the $i$-fold skew-suspension $\bar{S}^{i}(P, \theta)$ of the nonsingular $(-)^{i}$-quadratic form over $A$

$$
\begin{aligned}
(P, \theta)=\left(\operatorname { c o k e r } \left(\left(\begin{array}{cc}
d^{*} & 0 \\
(-)^{i+1}(1+T) \psi_{0} & d
\end{array}\right):\right.\right. & \\
& \left.\left.C^{i-1} \oplus C_{i+2} \rightarrow C^{i} \oplus C_{i+1}\right),\left[\begin{array}{cc}
\psi_{0} & d \\
0 & 0
\end{array}\right]\right)
\end{aligned}
$$

with $P$ a f.g. projective $A$-module. The error is repeated in Proposition 4.2.1iv) of Ranicki [16]. However, if $(C, \psi)$ is a $2 i$-dimensional quadratic Poincaré complex over any $A$ with f.g. projective homology $A$-modules $H_{*}(C)$ then Corollary 2.3 above shows that $(C, \psi) \in L_{2 i}^{p}(A)$ is represented by $\bar{S}^{i}\left(H^{i}(C), \psi\right)$. Thus the description of the isomorphism $L_{4 k+2}(Z) \stackrel{\sim}{\rightarrow} Z_{2}$ in Propositions 7.2 of [15, I] and 4.3.1 of [16] should read $(C, \psi) \rightarrow$ Arf invariant of $\left(H_{2 k+1}\left(C ; Z_{2}\right), \psi\right)$. For a $2 i$-dimensional symmetric Poincaré complex $(C, \phi)$ over a Dedekind ring $A$ the proof of Proposition 4.5 of $[15, \mathrm{I}]$ does show that $(C, \phi) \in L_{p}^{2 i}(A)$ is represented by $\bar{S}^{i}\left(F^{i}(C), \phi\right)$.

3. Some product formulae. We consider a product $(m+n)$-dimensional normal map

$$
g=1 \times f: M_{1}^{m} \times M^{n} \rightarrow M_{1}^{m} \times N^{n}
$$

with $M_{1}^{m}$ a closed $m$-dimensional manifold, $f: M^{n} \rightarrow N^{n}$ an $n$-dimensional normal map, and

$$
\pi_{1}\left(M_{1}\right)=\pi_{1}, \quad \pi_{1}(N)=\pi, \quad \pi_{1}\left(M_{1} \times N\right)=\pi_{1} \times \pi .
$$

The surgery obstruction $\sigma_{*}(g) \in L_{m+n}^{h}\left(Z\left[\pi_{1} \times \pi\right]\right)$ is determined algebraically by the symmetric signature $\sigma^{*}\left(M_{1}\right) \in L_{h}^{m}\left(Z\left[\pi_{1}\right]\right)$ and the surgery obstruction $\sigma_{*}(f) \in$ $L_{n}^{h}(Z[\pi])$; there is defined a product operation

$$
\begin{gathered}
L_{h}^{m}\left(Z\left[\pi_{1}\right]\right) \otimes L_{n}^{h}(Z[\pi]) \rightarrow L_{m+n}^{h}\left(Z\left[\pi_{1} \times \pi\right]\right) \\
(C, \phi) \otimes(D, \psi) \rightarrow\left(C \otimes_{Z} D, \phi \otimes \psi\right)
\end{gathered}
$$


which on the chain level is just the tensor product of chain complexes, and

$$
\sigma_{*}(g)=\sigma^{*}\left(M_{1}\right) \otimes \sigma_{*}(f) \in L_{m+n}\left(Z\left[\pi_{1} \times \pi\right]\right),
$$

where

$$
\sigma^{*}\left(M_{1}\right)=(C, \phi) \in L_{h}^{m}\left(Z\left[\pi_{1}\right]\right), \quad \sigma_{*}(f)=(D, \psi) \in L_{n}^{h}(Z[\pi])
$$

with $H_{*}(C)=H_{*}\left(\tilde{M}_{1}\right), H_{*}(D)=K_{*}(M)$; see Ranicki [15] for details.

It is not in general possible to evaluate (3.1) for finite groups $\pi_{1}, \pi$, since the symmetric $L$-groups $L^{*}\left(Z\left[\pi_{1}\right]\right)$ are comparable in size to the entire bordism groups $\Omega_{*}\left(B \pi_{1}\right)$ (by unpublished work of G. Carlsson, for example). So it is better to try and evaluate the particular products of (3.2) in the favorable circumstances where $\sigma_{*}(g)$ can be expressed in terms of the homology modules $H_{*}\left(\tilde{M}_{1}\right), K_{*}(M)$.

THEOREM 3.3. If $f: M^{n} \rightarrow N^{n}$ is a normal map such that

$$
\sigma_{*}(f)=\partial([P]) \in \operatorname{im}\left(\partial: \hat{H}_{n}\left(Z_{2} ; \tilde{K}_{0}(Z[\pi])\right) \rightarrow L_{n}^{h}(Z[\pi])\right)
$$

for some f.g. projective $Z[\pi]$-module $P$, then the induced f.g. projective $Z\left[\pi_{1} \times \pi\right]$ module $Z\left[\pi_{1}\right] \otimes_{Z} P$ is such that

$$
\begin{aligned}
\sigma_{*}(g) & =\chi\left(M_{1}\right) \partial\left(\left[Z\left[\pi_{1}\right] \otimes_{Z} P\right]\right) \\
& \in \operatorname{im}\left(\partial: \hat{H}_{m+n}\left(Z_{2}, \tilde{K}_{0}\left(Z\left[\pi_{1} \times \pi\right]\right)\right) \rightarrow L_{m+n}^{h}\left(Z\left[\pi_{1} \times \pi\right]\right)\right)
\end{aligned}
$$

with $\chi\left(M_{1}\right) \in Z$ the Euler characteristic of $M_{1}$. In particular, if $m$ is odd, then $\chi\left(M_{1}\right)=0$ and $\sigma_{*}(g)=0$.

Proof. Let $\left(C=C\left(\tilde{M}_{1}\right), \phi\right)$ be the symmetric Poincaré complex of $M_{1}$, and let $(D, \psi)$ be the quadratic Poincare kernel of $f$, so that

$$
\begin{gathered}
H_{*}(C)=H_{*}\left(\tilde{M}_{1}\right), \quad H_{*}(D)=K_{*}(M), \\
{[C]=\left[C\left(\tilde{M}_{1}\right)\right]=\chi\left(M_{1}\right)\left[Z\left[\pi_{1}\right]\right] \in K_{0}\left(Z\left[\pi_{1}\right]\right) \quad \text { (unreduced). }}
\end{gathered}
$$

By Remark $2.2(D, \psi)$ admits a projective null-cobordism $(\delta D, D ; \delta \psi)$ with $[\delta D]=$ $[P] \in \tilde{K}_{0}(Z[\pi])$. Then $(C, \phi) \otimes(D, \psi)$ admits a projective null-cobordism $(C \otimes$ $\delta D, C \otimes D ; \phi \otimes \delta \psi)$ such that

$$
[C \otimes \delta D]=\left[C\left(\tilde{M}_{1}\right) \otimes \delta D\right]=\chi\left(M_{1}\right)\left[Z\left[\pi_{1}\right] \otimes_{Z} P\right] \in \tilde{K}_{0}\left(Z\left[\pi_{1} \times \pi\right]\right),
$$

and the theorem follows from Remark 2.2.

The product of (3.1) has a version for the torsion $L$-groups

$$
\begin{gathered}
L_{h}^{m}\left(Z\left[\pi_{1}\right]\right) \otimes L_{n+1}^{h, \text { tor }}(Z[\pi]) \rightarrow L_{m+n+1}^{h, \text { tor }}\left(Z\left[\pi_{1} \times \pi\right]\right) ; \\
(C, \phi) \otimes(D, \psi) \rightarrow(C \otimes D, \phi \otimes \psi),
\end{gathered}
$$

so that if

$$
\sigma_{*}(f) \in \operatorname{im}\left(L_{n+1}^{h, \operatorname{tor}}(Z[\pi]) \rightarrow L_{n}^{h}(Z[\pi])\right),
$$

then

$$
\sigma_{*}(g) \in \operatorname{im}\left(L_{m+n+1}^{h, \text { tor }}\left(Z\left[\pi_{1} \times \pi\right]\right) \rightarrow L_{m+n}^{h}\left(Z\left[\pi_{1} \times \pi\right]\right)\right) .
$$


THEOREM 3.4. Let $n=\operatorname{dim} N=2 i+1$ be odd in (3.1), and suppose that $\sigma_{*}(f) \in$ $L_{2 i+1}^{h}(Z[\pi])$ is the image of $(V, \lambda, \mu) \in L_{2 i+2}^{h \cdot t o r}(Z[\pi])$ with $(V, \lambda, \mu)$ a nonsingular $(-)^{i+1}$-quadratic linking form on an FPL 1 torsion $Z[\pi]$-module $V$ such that $(|V|$, $\left.\left|\pi_{1} \times \pi\right|\right)=1$ (assuming $\pi_{1}$ and $\pi$ are finite), and such that $|V|$ is also prime to all the torsion in $H_{*}\left(\tilde{M}_{1}\right)$. Then

(a) if $m=\operatorname{dim} M_{1}=2 j+1$,

$$
\begin{aligned}
\sigma_{*}(g) & =\partial\left(\sum_{k=0}^{j}(-)^{k} \chi\left(H_{k}\left(\tilde{M}_{1}\right) \otimes V\right)\right) \\
& \in \operatorname{im}\left(\partial: \hat{H}_{2 i+2 j+2}\left(Z_{2}, \tilde{K}_{0}\left(Z\left[\pi_{1} \times \pi\right]\right)\right) \rightarrow L_{2 i+2 j+2}^{h}\left(Z\left[\pi_{1} \times \pi\right]\right)\right),
\end{aligned}
$$

(b) if $m=2 j, \sigma_{*}(g) \in L_{2 i+2 j+1}^{h}\left(Z\left[\pi_{1} \times \pi\right]\right)$ is the image of

$$
\left(H_{j}\left(\tilde{M}_{1}\right) \otimes V, \phi \otimes(\lambda, \mu)\right) \oplus H_{(-)^{\prime+1+1}}^{\text {tor }}(L) \in L_{2 i+2 j+2}^{h, \text { tor }}\left(Z\left[\pi_{1} \times \pi\right]\right)
$$

with $L$ a PL 1 torsion $Z\left[\pi_{1} \times \pi\right]$-module such that

$$
\chi(L)=(-)^{j} \sum_{k=0}^{j-1}(-)^{k} \chi\left(H_{k}\left(\tilde{M}_{1}\right) \otimes V\right) \in \tilde{K}_{0}\left(Z\left[\pi_{1} \times \pi\right]\right) .
$$

In particular, if $H_{j}\left(\tilde{M}_{1}\right)=0$, then

$$
\begin{aligned}
\sigma_{*}(g) & =\partial\left(\sum_{k=0}^{j-1}(-)^{k} \chi\left(H_{k}\left(\tilde{M}_{1}\right) \otimes V\right)\right) \\
& \in \operatorname{im}\left(\partial: \hat{H}_{2 i+2 j+1}\left(Z_{2}, \tilde{K}_{0}\left(Z\left[\pi_{1} \times \pi\right]\right)\right) \rightarrow L_{2 i+2 j+1}^{h}\left(Z\left[\pi_{1} \times \pi\right]\right)\right) .
\end{aligned}
$$

PROOF. By surgery below the middle dimension we may assume that

$$
K_{r}(M)=\left(\begin{array}{ll}
V & \text { if } r=i \\
0 & \text { if } r \neq i
\end{array}\right.
$$

It follows by the Künneth theorem and the assumption on $V$ that

$$
K_{r}\left(M_{1} \times M\right)=\left(\begin{array}{ll}
0 & \text { if } r<i \text { or } r>i+m, \\
H_{r-i}\left(\tilde{M}_{1}\right) \otimes V & \text { if } i \leqslant r \leqslant i+m,
\end{array}\right.
$$

so that $g=1 \times f: M_{1} \times M \rightarrow M_{1} \times N$ has PL 1 torsion kernel $Z\left[\pi_{1} \times \pi\right]$-modules $K_{*}\left(M_{1} \times M\right)$ (cf. Remark 1.5). Moreover, if $m+n=4 k+2$, the Kervaire invariant $\sigma_{*}(g) \in L_{m+n}(Z)=Z_{2}$ is 0 , since it is the evaluation on $\sigma^{*}\left(M_{1}\right) \otimes \sigma_{*}(f)$ of the product $L^{m}(Z) \otimes L_{n}(Z) \rightarrow L_{m+n}(Z)$ (a special case of (3.1)) and $\sigma_{*}(f) \in$ $L_{n}(Z)=L_{2 i+1}(Z)=0$. The theorem now follows by a direct appliction of Theorems $1.11,1.14$.

EXAMPLE 3.5. We start with the surgery problem

$$
\text { id } \times f: \mathbf{R} \mathbf{P}^{4 i+1} \times K^{4 j+2} \rightarrow \mathbf{R} \mathbf{P}^{4 i+1} \times S^{4 j+2}
$$

where $f$ represents the usual Kervaire problem. Then Wall has proved [20, Theorem $13 B .7$, p. 181] that this problem represents the nontrivial element in the surgery group $L_{3}^{h}(Z / 2)=Z / 2$. Hence (3.6) can be surgered to

$$
g: N^{4(i+j)+3} \rightarrow \mathbf{R P}^{4 i+1} \times S^{4 j+2}
$$


with

$$
K_{*}(g)=\left(\begin{array}{ll}
0 & \text { if } * \neq 2(i+j)+1 \\
Z /(8 k+3) & \text { with trivial } Z / 2 \text { action, } 8 k+3 \text { prime if } *=2(i+j)+1
\end{array}\right.
$$

Consequently, we have that

$$
\text { id } \times \text { id } \times f: \mathbf{R P}^{4 s \pm 1} \times \mathbf{R} \mathbf{P}^{4 i+1} \times K^{4 j+2} \rightarrow \mathbf{R} \mathbf{P}^{4 s \pm 1} \times \mathbf{R} \mathbf{P}^{4 i+1} \times S^{4 j+2}
$$

is represented by $\partial\{Z /(8 k+3)\}$. But since $\tilde{K}_{0}(Z / 2 \times Z / 2)=0$, it follows that $\sigma_{*}(\mathrm{id} \times$ id $\times f)=0$ in all these cases.

4. A nontrivial example and other applications. We shall now construct a family of products with nontrivial surgery obstructions. The first of these examples was discovered by J. Morgan and W. Pardon, and later analyzed by L. Taylor and B. Williams using different techniques.

Let $\pi=Z / 4 \times Z / 2$, with generators $t \in Z / 2, u \in Z / 4$. Define a 4-dimensional normal map of closed manifolds

$$
g=1 \times f: T^{2} \times T^{2} \rightarrow T^{2} \times S^{2}
$$

with $f: T^{2} \rightarrow S^{2}$ the 2-dimensional normal map with Kervaire invariant

$$
\sigma_{*}(f)=1 \in L_{2}(Z)=Z_{2} .
$$

The original Morgan-Pardon example is the element $\sigma_{*}(g) \in L_{4}^{h}(\pi)$ obtained from the actual surgery obstruction $\sigma_{*}(g) \in L_{4}^{h}(Z \times Z)$ via the evident surjection of groups

$$
\pi_{1}\left(T^{2}\right)=Z \times Z=\langle T, U \mid T U=U T\rangle \rightarrow \pi ; \quad T \rightarrow t, U \rightarrow u .
$$

In fact, this example turns out to be a special case of the product formula, which we will apply in Corollary 4.10 to obtain the complete answer for this group.

The following result provides a nice example of the use of the torsion projective semicharacteristic (Theorem 1.11).

Proposition 4.1. The element $\sigma_{*}(g) \in L_{4}^{h}(\pi)$ is the image under $\partial$ of the nontrivial element $[P] \in \hat{H}_{0}\left(Z_{2} ; \tilde{K}_{0}(Z[\pi])\right)=Z / 2$ represented by the $P L 1$ odd torsion $Z[\pi]-$ module

$$
P=Z[\pi] /(2-t, 1-u)=Z / 3,
$$

that is $\sigma_{*}(g)=\partial([P]) \in L_{4}^{h}(\pi)$.

Proof. Define rings

$$
\begin{aligned}
\Lambda & =Z(Z)=Z\left[T, T^{-1}\right], \\
\Lambda^{\prime} & =\Lambda \otimes \Lambda=Z(Z \times Z)=Z\left[T, T^{-1}, U, U^{-1}\right],
\end{aligned}
$$

with the involutions $\bar{T}=T^{-1}, \bar{U}=U^{-1}$. The surgery obstruction $\sigma_{*}(g) \in L_{4}^{h}\left(\Lambda^{\prime}\right)$ is represented by the 4-dimensional quadratic Poincaré complex over $\Lambda^{\prime}$

$$
\sigma_{*}(g)=\sigma^{*}\left(S^{1}\right) \otimes \sigma^{*}\left(S^{1}\right) \otimes \sigma_{*}(f),
$$


with $\sigma^{*}\left(S^{1}\right)=(C, \phi)$ the 1-dimensional symmetric Poincare complex over $\Lambda$ of the circle $S^{1}$ and $\sigma_{*}(f)=(D, \psi)$ the 2-dimensional quadratic Poincaré kernel over $Z$ of $f$,

$$
\begin{gathered}
d=1-T: C_{1}=\Lambda \rightarrow C_{0}=\Lambda, \\
\phi_{0}=\left(\begin{array}{c}
1: C^{0} \rightarrow C_{1}, \\
-T: C^{1} \rightarrow C_{0},
\end{array} \quad \phi_{1}=1: C^{1} \rightarrow C_{1},\right. \\
\psi_{0}=\left(\begin{array}{ll}
1 & 1 \\
0 & 1
\end{array}\right): D^{1}=Z \oplus Z \rightarrow D_{1}=Z \oplus Z, \quad D_{0}=D_{2}=0 .
\end{gathered}
$$

The product

$$
\sigma^{*}\left(S^{1}\right) \otimes \sigma_{*}(f)=(C \otimes D, \phi \otimes \psi)
$$

is a 3-dimensional quadratic Poincaré complex over $\Lambda$ with homology $\Lambda$-modules

$$
H_{r}(C \otimes D)=\left(\begin{array}{ll}
Z \oplus Z, & r=1, \\
0, & r \neq 1 .
\end{array}\right.
$$

Algebraic surgery on the two $\Lambda$-module generators of $H_{1}(C \otimes D)$ results in a cobordant 3-dimensional quadratic Poincaré complex $(E, \theta)$ over $\Lambda$ with

$$
H_{r}(E)=\left(\begin{array}{ll}
\Lambda /\left(1-T+T^{2}\right), & r=1, \\
0, & r \neq 1,
\end{array}\right.
$$

where

$$
1-T+T^{2}=\left|\begin{array}{cc}
1-T & 1 \\
-T & 1-T
\end{array}\right|
$$

( = the Alexander polynomial of the trefoil knot). Thus

$$
(C, \phi) \otimes(D, \psi)=(E, \theta) \in L_{3}^{h}(\Lambda)
$$

and $\sigma_{*}(g)=(C, \phi) \otimes(C, \phi) \otimes(D, \psi)$ is cobordant to the 4-dimensional quadratic Poincaré complex over $\Lambda^{\prime}\left(E^{\prime}, \theta^{\prime}\right)=(C, \phi) \otimes(E, \theta)$ with $\Lambda^{\prime}$-homology modules

$$
H_{r}\left(E^{\prime}\right)=\left(\begin{array}{ll}
\Lambda /\left(1-T+T^{2}, 1-U\right), & r=1,2, \\
0, & r \neq 1,2 .
\end{array}\right.
$$

Changing the coefficient ring from $\Lambda^{\prime}$ to $Z[\pi]$ by the surjection $\Lambda^{\prime} \rightarrow Z[\pi] ; T \rightarrow t$, $U \rightarrow u$ we have

$$
H_{r}\left(Z[\pi] \otimes_{\Lambda^{\prime}} E^{\prime}\right)=\left(\begin{array}{ll}
Z[\pi] /(2-t, 1-u)=P, & r=1,2, \\
0, & r \neq 1,2,
\end{array}\right.
$$

so that by Theorem 1.11

$$
\begin{aligned}
\sigma_{*}(g) & =Z[\pi] \otimes_{\Lambda^{\prime}}\left(E^{\prime}, \theta^{\prime}\right) \\
& =\partial([P]) \in \operatorname{im}\left(\partial: \hat{H}_{0}\left(Z_{2} ; \tilde{K}_{0}(Z[\pi])\right) \rightarrow L_{4}^{h}(\pi)\right) .
\end{aligned}
$$

LEMMA 4.2. $\tilde{K}_{0}(Z[\pi])=Z / 2$ with explicit representative generated by $\chi[Z(i) /(1-2 i)]=\chi\left(\left\langle Z^{++} / 3\right\rangle\right)=\chi\left(\left\langle Z^{+-} / 3\right\rangle\right)=\chi\left(\left\langle Z^{-+} / 3\right\rangle\right)=\chi\left(\left\langle Z^{--} / 3\right\rangle\right)$. 
Proof. We assume it is well known that $\tilde{K}_{0}(Z[Z / 2 \times Z / 2])=0$. There is an exact sequence

$$
K_{1}(Z \pi) \rightarrow K_{1}\left(Z\left(\frac{1}{2}\right) \pi\right) \oplus K_{1}\left(\hat{Z}_{2} \pi\right) \rightarrow K_{1}\left(\hat{Q}_{2} \pi\right) \rightarrow \tilde{K}_{0}(Z \pi) \rightarrow 0
$$

(see e.g. Hambleton-Milgram [9]), and

$$
\begin{aligned}
K_{1}\left(\hat{Q}_{2} \pi\right) / K_{1}\left(Z\left(\frac{1}{2}\right) \pi\right) \oplus \operatorname{im}(1+ & \left.I\left\{\hat{Z}_{2}(\pi)\right\}\right) \\
& \cong \frac{U\left(\hat{Z}_{2}(i)^{+}\right) \oplus U\left(\hat{Z}_{2}(i)^{-}\right) \oplus\left(\hat{Z}_{2}\right)^{4}}{\left\langle 1+8 u_{1}\right\rangle \oplus\left\langle 1+8 u_{2}\right\rangle \oplus \cdots \oplus\left\langle 1+8 u_{6}\right\rangle} .
\end{aligned}
$$

To obtain the rest of the calculation, consider the exact sequence which calculates $K_{1}\left(\hat{Z}_{2}(\pi)\right)$,

$$
\begin{aligned}
0 \rightarrow K_{1}\left(\hat{Z}_{2} \pi .\right) \rightarrow K_{1}\left(\hat{Z}_{2}(Z / 2 \times Z / 2)\right) \oplus K_{1}\left(\hat{Z}_{2}(i)[Z / 2]\right) \\
\rightarrow K_{1}\left(F_{2}(Z / 2 \times Z / 2)\right) .
\end{aligned}
$$

Here $F_{2}(Z / 2 \times Z / 2)^{*}=(Z / 2)^{3}$ with generators $u, t, 1+u+t$ (where $t, u$ are generators for $Z / 2 \times Z / 2)$. The first two are cancelled from $K_{1}\left(Z\left(\frac{1}{2}\right) \pi\right)$, but the third is not and forces the $Z / 2$ in $\tilde{K}_{0}(\pi)$. To see that this element $\chi\left(\langle 3\rangle^{++}\right)$is also represented by the other elements claimed in 4.2 consider the unit $1+(t-1) u$ $\left(t^{2}=1, u^{4}=1\right)$ in $\hat{Z}_{2}(\pi)$. Its images are

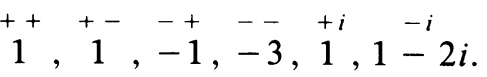

Next consider $1+(t-1)\left(u^{2}+1\right)$ with image

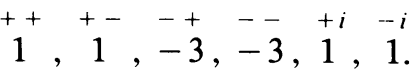

Also, $u\left(1-u+u^{2}\right)$ has image

$$
\stackrel{++}{1},-3,-1^{+},--3,1,-i
$$

and finally, $1-(t+1)\left(u^{2}+1\right)$ has image

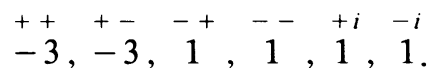

This completes the proof.

COROLLARY 4.4. The $L^{h}$-groups of $\pi=Z / 4 \times Z / 2$ are given by

$$
L_{*}^{h}(\pi)=\left(\begin{array}{ll}
Z^{6} \oplus Z / 2, & *=0, \\
Z / 2, & *=1, \\
(Z / 2) \oplus Z^{2}, & *=2, \\
(Z / 2)^{3}, & *=3
\end{array}\right.
$$

In particular, $L_{3}^{h}(\pi) \rightarrow L_{3}^{p}(\pi)$ is an injection and

$$
0 \rightarrow\left\{\hat{H}_{0}\left(Z / 2, \tilde{K}_{0}(\pi)\right)=Z / 2\right\} \stackrel{\partial}{\rightarrow} L_{0}^{h}(\pi)
$$

is injective. 
Proof. Using Hambleton-Milgram [9, Lemmas 5.4, 5.7] and 4.2 we have that the compositions

$$
\begin{aligned}
& L_{0}^{p}(\pi) \rightarrow Z^{6} \rightarrow \hat{H}_{\text {odd }}\left(Z / 2, \tilde{K}_{0}(\pi)\right), \\
& L_{2}^{p}(\pi) \rightarrow Z^{2} \rightarrow \hat{H}_{\text {odd }}\left(Z / 2, \tilde{K}_{0}(\pi)\right)
\end{aligned}
$$

are both zero. (This is an exceptional case, normally the map would have been onto, but $Z[i]$ does not have any nontorsion units.) But $L_{3}^{p}(\pi) \rightarrow \hat{H}_{\mathrm{ev}}\left(Z / 2, \tilde{K}_{0}(\pi)\right)$ is onto, and since $L_{1}^{p}(\pi)=0$, the result follows.

THEOREM 4.6. The elements in $L_{*}^{h}(\pi)$ which are detected by surgery problems on closed manifolds are $Z \oplus Z / 2 \subset L_{0}^{h}(\pi)$ coming from the simply connected index obstruction, and the Morgan-Pardon example, $Z / 2 \subset L_{2}^{h}(\pi)$ (the simply connected Kervaire problem) and $(Z / 2)^{2} \subset L_{3}^{h}(\pi)$.

Remark 4.7. The Morgan-Pardon surgery problem is the simplest example of a nontrivial obstruction in the image of the map $\partial$ in (4.5).

Proof of 4.6. Since $\Omega_{*}(\mathrm{pt}) \otimes Q \rightarrow \Omega_{*}(\pi) \otimes Q$ is an isomorphism, it follows that the only $Z$-free classes which occur have some finite multiples in the image of $L_{*}(1)$. Thus the only thing left to prove is that the extra class in $L_{0}^{h}(\pi)$ also detects.

Consider the surgery problem

$$
\text { id } \times p: L_{4}^{5} \times \mathbf{R} \mathbf{P}^{5} \times K^{6} \rightarrow L_{4}^{5} \times \mathbf{R} \mathbf{P}^{5} \times S^{6} .
$$

Doing surgery on $\mathbf{R P}^{5} \times K^{6}$, we can assume (4.8) equivalent to

$$
\text { id } \times p^{\prime}: L_{4}^{5} \times M^{\prime \prime} \rightarrow L_{4}^{5} \times S^{6}
$$

with $K_{*}\left(p^{\prime}\right)=K_{5}\left(p^{\prime}\right)=(Z / 3)^{++}$. Then applying 3.3, we have

$$
\sigma_{*}\left(\operatorname{id} \times p^{\prime}\right)=\partial\left\{\chi\left(Z^{+} \otimes(Z / 3)^{+}\right)\right\}=\partial\left\{\chi\left(Z / 3^{++}\right)\right\},
$$

and the result follows from 4.2.

Corollary 4.10. Let $\pi_{i}=Z / 2^{i} \times Z / 2, i \geqslant 2$.

(a) $\operatorname{im}\left(\partial: \hat{H}_{\mathrm{ev}}\left(Z / 2, \tilde{K}_{0}\left(\pi_{1}\right)\right) \rightarrow L_{0}^{h}\left(\pi_{i}\right)\right)$ is never 0 .

(b) There is a closed 4i-dimensional manifold $N$ with fundamental group $\pi_{i}$ and a surgery problem $f: M \rightarrow N$ with nontrivial $L^{h}$-surgery obstruction in the image of $\partial$.

Proof. The problem

$$
\text { id } \times p: L_{2^{i}}^{5} \times \mathbf{R} \mathbf{P}^{5} \times K^{4 j+2} \rightarrow L_{2^{i}}^{5} \times \mathbf{R} \mathbf{P}^{5} \times S^{4 j+2}
$$

will serve since a covering of this problem is the one used in the proof of 4.6 . Moreover, the index invariant of this problem is zero, so the image of the surgery obstruction in $L_{0}^{p}\left(\pi_{i}\right)=Z^{\nu(i)}$ is 0 , and the result follows.

REMARK 4.11. This process ends when we go to 3-fold products. Indeed, Theorem 3.3 shows that the surgery obstruction

$$
\text { id } \times p: L_{2^{\prime}} \times L_{2^{j}} \times L_{2^{k}} \times K^{4 s+2} \rightarrow L_{2^{\prime}} \times L_{2^{\prime}} \times L_{2^{k}} \times S^{4 s+2}
$$

is always 0 . 
In fact one of us will show in [10] that the Morgan-Pardon example is, in a very precise sense, the only iterated product that can occur for surgery problems with finite fundamental group. Indeed, [10], building on the work initiated here will show that the only possible obstructions in these cases occur from products $\mathbf{R P}^{4 i+1}$, situations induced up from Morgan-Pardon examples, and situations induced up from Cappell-Shaneson examples.

However, the structure of product formulae is much richer when we look at infinite groups. Indeed, both the Milnor and Kervaire problems can be producted arbitrarily often with circles to obtain nontrivial surgery problems in $L_{n}^{h}\left(Z\left[Z^{k}\right]\right)$.

\section{REFERENCES}

1. A. Bak and M. Kolster, The computation of odd-dimensional projective surgery groups of finite groups, Topology 21 (1982), 35-63.

2. S. Cappell and J. Shaneson, A counterexample on the oozing conjecture, (Proc. 1978 Aarhus Topology Conf), Lecture Notes in Math., vol. 763, Springer-Verlag, New York, 1979, pp. 627-634.

3. G. Carlesson and J. Milgram, The structure of odd L-groups, (Proc. Waterloo Conf. on Algebraic Topology), Lecture Notes in Math., vol. 741, Springer-Verlag, New York, 1979, pp. 1-72.

4. , Some exact sequences in the theory of hermitian forms, J. Pure Appl. Algebra 18 (1980), $233-252$.

5. The oriented odd L-groups of finite groups (preprint).

6. F. Clauwens, The K-theory of almost symmetric forms, Topological Structures II, Math. Centre Tracts, no. 115, Math. Centrum, Amsterdam, 1979, pp. 41-49.

7. J. F. Davis, The surgery semicharacteristic, Proc. London Math. Soc. (3) 47 (1983), 411-428.

8. I. Hambleton, Projective surgery obstructions on closed manifolds, (Proc. 1980 Oberwolfach Conf. on Algebraic K-theory), Lecture Notes in Math., vol. 967, Springer-Verlag, New York, 1982, pp. 101-131.

9. I. Hambleton and J. Milgram, The surgery obstruction groups for finite 2-groups, Invent. Math. 61 (1980), 33-52.

10. J. Milgram, Surgery with finite fundamental group I: The obstructions (to appear).

11. W. Pardon, Local surgery and the exact sequence of a localization for Witt groups, Mem. Amer. Math. Soc. No. 196 (1977).

12. The exact sequence of a localization for Witt groups II. Numerical invariants for odd-dimensional surgery obstructions, Pacific J. Math. 102 (1982), 123-169.

13. A. A. Ranicki, Algebraic L-theory I. Foundations, Proc. London Math. Soc. (3) 27 (1973), 101-125.

14. On the algebraic L-theory of semisimple rings, J. Algebra 50 (1978), 242-243.

15. The algebraic theory of surgery. I, II, Proc. London Math. Soc. (3) 40 (1980), 87-192; 40 (1980), 193-283.

16. Exact sequences in the algebraic theory of surgery, Math. Notes 26, Princeton Univ. Press, Princeton, N. J., 1981.

17. C. P. Rourke and D. P. Sullivan, On the Kervaire obstruction, Ann. of Math. (2) 94 (1971), 397-413.

18. L. Taylor and B. Williams, Surgery on closed manifolds (preprint).

19. C. T. C. Wall, Surgery on non-simply-connected manifolds, Ann. of Math. (2) 84 (1966), 217-276.

20. __ Surgery on compact manifolds, Academic Press, New York, 1970.

21. Classification of hermitian forms VI. Group rings, Ann. of Math. (2) 103 (1976), 1-80.

22. J. F. Davis and A. A. Ranicki, Semi-invariants in surgery (preprint)

Department of Mathematics, Stanford University, Stanford, California 94305

Department of Mathematics, Edinburgh University, Edinburgh, Scotland 